\title{
Article \\ Optimized Application of Sustainable Development Strategy in International Engineering Project Management
}

\author{
Zhiwu Zhou (D), Julián Alcalá and Víctor Yepes *(D) \\ Institute of Concrete Science and Technology (ICITECH), Universitat Politècnica de València, \\ 46022 Valencia, Spain; zhizh2@doctor.upv.es (Z.Z.); jualgon@cst.upv.es (J.A.) \\ * Correspondence: vyepesp@cst.upv.es; Tel.: +34-96-387-9563
}

check for updates

Citation: Zhou, Z.; Alcalá, J.; Yepes,

V. Optimized Application of

Sustainable Development Strategy in International Engineering Project Management. Mathematics 2021, 9 , 1633. https://doi.org/10.3390/ math9141633

Academic Editor: Giampaolo Liuzzi

Received: 31 May 2021

Accepted: 2 July 2021

Published: 10 July 2021

Publisher's Note: MDPI stays neutral with regard to jurisdictional claims in published maps and institutional affiliations.

Copyright: (C) 2021 by the authors. Licensee MDPI, Basel, Switzerland. This article is an open access article distributed under the terms and conditions of the Creative Commons Attribution (CC BY) license (https:/ / creativecommons.org/licenses/by/ $4.0 /)$.

\begin{abstract}
The aim of this paper is to establish an international framework for sustainable project management in engineering, to make up the lack of research in this field, and to propose a scientific theoretical basis for the establishment of a new project management system. The article adopts literature review, mathematical programming algorithm and case study as the research method. The literature review applied the visual clustering research method and analyzed the results of 21-year research in this field. As a result, the project management system was found to have defects and deficiencies. A mathematical model was established to analyze the composition and elements of the optimized international project management system. The case study research selected large bridges for analysis and verified the superiority and practicability of the theoretical system. Thus, the goal of sustainable development of bridges was achieved. The value of this re-search lies in establishing a comprehensive international project management system model; truly integrating sustainable development with project management; providing new research frames and management models to promote the sustainable development of the construction industry.
\end{abstract}

Keywords: bridge; project management; environmental impact; cost; optimization

\section{Introduction}

Global warming is threatening the survival of humans and other species [1]. In response to severe environmental crises and global development inequality, sustainable development strategies have been implemented. It can meet the needs of the present with-out compromising the needs of the next generation [2].

Wang et al. [3] find that China accounted for $21 \%$ of global energy consumption, surpassing the United States and becoming the world's largest emitter of carbon dioxide $\left(\mathrm{CO}_{2}\right)$. The construction industry is one of the 12 key sectors making significant contributions to the $\mathrm{CO}_{2}$ emissions from 2005 to 2020. According to Liu et al. [4], China's construction industry accounted for $64.6 \%$ of total emissions from 1991 to 2010 , with an average annual growth rate of $5.7 \%$, and carbon emissions will approach the peak level by 2030 and to achieve the goal of environmental control.

Zhou et al. [5] concluded that the environmental impact range of the bridge construction stage is $6.3 \sim 34.09 \%$. It is therefore a question of reducing the overexploitation of fossil energy, high-cost risks and excessive pollution problems [6]. Regeneration and utilization of clean energy, promotion of sustainable building development and other issues have become the main options facing scientific researchers, national government agencies and international organizations around the world $[7,8]$.

This study attempts to analyze the reasons for differences in the value of environmental pollution: materials; machinery; project management personnel; construction methods; and factors in the construction environment. The objective of this paper is to find out the relationship between project management and the impact of sustainable development. In addition, the purpose is to optimize project management to achieve sustainable development objectives. 


\section{Literature Review}

This study analyzed 7753 articles published on sustainable development and project management between 2000 and 2021 were selected. The Scopus database [9] and the analysis software Citespace [10] were used.

\subsection{Visual Clustering Coupling Analysis}

Figure 1 shows the keyword clustering network map. The average cluster contour value, $\mathrm{S}=0.7371>0.7$, indicates that the clustering is convincing. The map has the following characteristics: number of nodes $\mathrm{N}=926$; number of connections $\mathrm{E}=4370$; and net-work modular clustering index $Q=0.4469$. This show that the quality of this clustering is at intermediate level $(0<\mathrm{Q}<1)$. The cooperation density of the research institution that publishes the article equals 0.0102 . A small value indicates that the research results are completed by independent institutions, and the rate of cooperative research between two or more institutions is low. The total citation frequency of more than two articles is CC $=903$, which accounts for the total citation frequency $97 \%$. The modularity index QS $=0.5564$, indicating a good structure in the clustering network. The keyword ranking after cluster analysis in 21 years is Physical activity $>$ Sustainable design $>$ Rural development project $>$ Renewable energy project $>$ Groundwater resource $>$ Sustainable development $>$ Multiple benefit $>$ Municipal solid waste $>$ Maternal health intervention. No management phrases were found in the nine key words, indicating that project management and sustainable development research is lacking. After 2013, with the increase in climate, social capital, management, etc., the types of research directions have improved. Research on project management and environmental sustainability have been extended to other disciplines, and the peak node of environmental sustainability research has shifted to multi-field development and research related to the construction industry, so it is necessary to strengthen and in-depth such research directions.

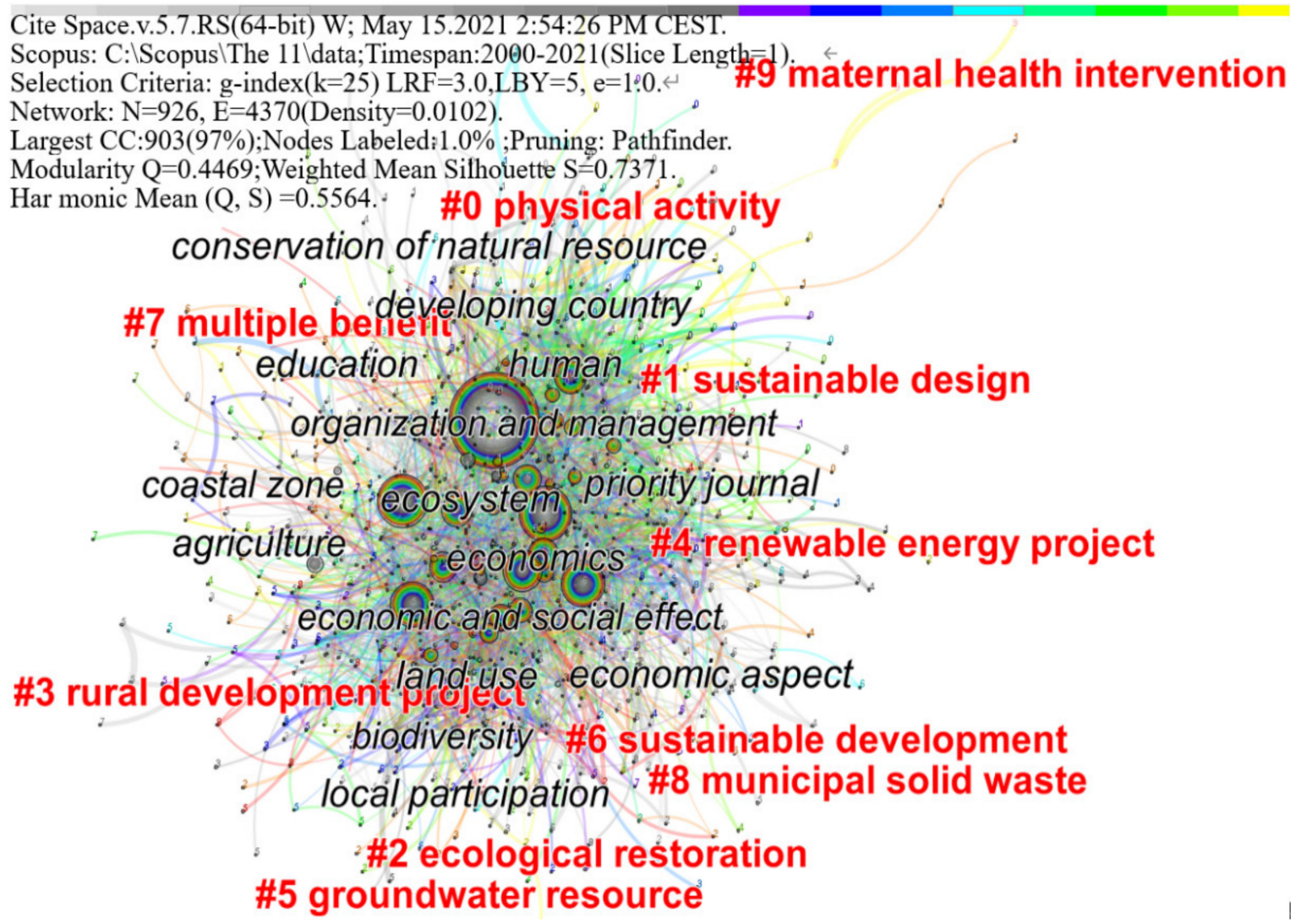

Figure 1. Keyword clustering network graph analysis of the survey literature (2000-2021). 
The occurrence of keywords during the period (2000-2021) is shown in Figure 2. The strength range ranks Risk Perception is 156.76 (2004 2009); Project Management is 112.03 (2000 2007), Engineering Management is concentrated in this stage; Human is 70.41 (2004 2009); Strategic Planning is 68.57 (2000 2010); Energy Efficiency is 59.12 (2004 2009).

\section{Top 25 Keywords with the Strongest Citation Bursts}

\begin{tabular}{|c|c|}
\hline \multicolumn{2}{|l|}{ Keywords } \\
\hline project management & 2000 \\
\hline strategic planning & 2000 \\
\hline societies and institution & 2000 \\
\hline economic and social effect & 2000 \\
\hline industrial management & 2000 \\
\hline public policy & 2000 \\
\hline \multicolumn{2}{|c|}{ research and development management 2000} \\
\hline industrial economics & 2000 \\
\hline environmental impact & 2000 \\
\hline social aspect & 2000 \\
\hline far east & 2000 \\
\hline mathematical model & 2000 \\
\hline ecological compensation & 2000 \\
\hline risk perception & 2000 \\
\hline construction industry & 2000 \\
\hline Habitat quality & 2000 \\
\hline brazil & 2000 \\
\hline eastern hemisphere & 2000 \\
\hline sub saharan africa & 2000 \\
\hline management & 2000 \\
\hline climate & 2000 \\
\hline Industrial management & 2000 \\
\hline research & 2000 \\
\hline procedure & 2000 \\
\hline sustainable development goal & 2000 \\
\hline
\end{tabular}

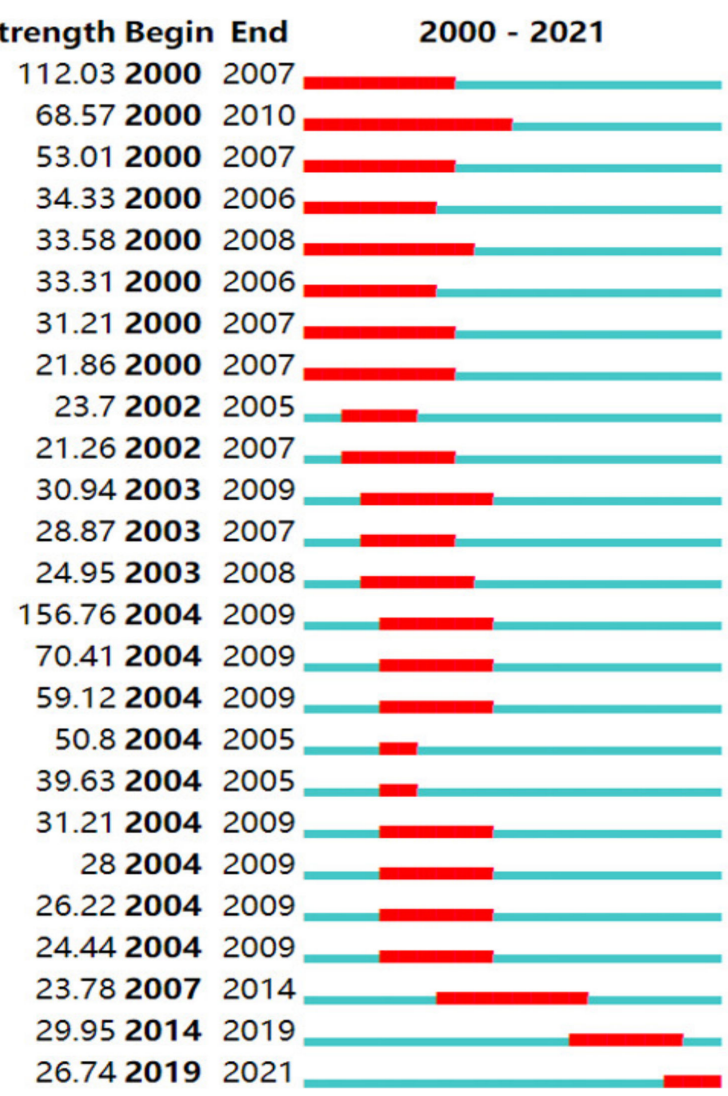

Figure 2. Strongest citation bursts chart analysis of survey statistics (which mainly explain the hot topics studied in the concentrated time interval).

In response to the preliminary findings of the overall cluster analysis, the scope of the keyword research was further narrowed, with an emphasis on individual article analysis and comparative research. Sustainable Project Management and Building was selected for the search and 1749 published articles were retrieved. Second, Bridge was selected as a keyword to retrieve 120 articles on sustainable development and project management related to bridges and the structure and scientific contribution of each article was analyzed in groups. The 23 most representative articles were selected, as shown in Table 1. In addition, an analysis of the future progress in this field is shown in Figure 2.

Figure 3a shows 31 (1990 2021) keyword cluster analysis in the year, the top four rankings of highly cited Strength are respectively (1) = Research is $27.85>$ (2) = Project management is $22.09>$ (3) = Procedure is $14.47>$ (4) = Societies and institution is 11.58. Analysis data shows that the research scope of 2012 2021 tends to the scope of survey, Architectural design, Design/methodology/approach, and adult, and the project management research results are lacking. Figure $3 \mathrm{~b}$ shows the results of cluster analysis of institutions and countries. The ranking of highly cited countries (4) $=$ USA is $6.31>$ (5) = United Kingdom is $6.21>$ (6) = China is $5.23>$ (7) $=$ Netherlands is $4.5>$ (8) = Spain is 4.36 . 
Table 1. Analysis and limitations of the highly relevant articles selected.

\begin{tabular}{|c|c|}
\hline References & Limitation (Analysis of the Representative Articles) \\
\hline [11] & $\begin{array}{l}\text { The research is mainly based on questionnaires, without the establishment of a systematic framework and model, } \\
\text { and lacks data analysis. }\end{array}$ \\
\hline [12] & $\begin{array}{l}\text { Restricted data research leads to limited availability, questionnaire surveys in a specific institutional context affect } \\
\text { the generalization of results, and the discussion mechanism is not sound. }\end{array}$ \\
\hline [13] & $\begin{array}{l}\text { The application of an integrated project delivery centralized cost management system needs to be proved by } \\
\text { research. }\end{array}$ \\
\hline [14] & $\begin{array}{l}\text { Data analysis is one-sided, and the use of Ghanaian construction industry data can ensure the success of projects in } \\
\text { other developing countries. }\end{array}$ \\
\hline [15] & $\begin{array}{l}\text { Used } 4 \mathrm{D} \text { bridge information management model technology for bridge monitoring. Explained a method of project } \\
\text { management without specific indicators, data, and framework standards for assessment. }\end{array}$ \\
\hline [16] & $\begin{array}{l}\text { Extending the results to other research areas may not effectively minimize the environmental hazards, and the } \\
\text { standard and standard weight are uncertain. Inherent human prejudice and subjective judgment system theory. }\end{array}$ \\
\hline [17] & $\begin{array}{l}\text { To identify and overcome the scarcity of elements, we need to develop a complete green bridge rating system and } \\
\text { theoretical research framework. }\end{array}$ \\
\hline [18] & $\begin{array}{l}\text { Due to the particularity and complexity of construction projects, there is no systematic research framework, so it is } \\
\text { difficult to promote. }\end{array}$ \\
\hline [19] & The specific research and analysis process is very clear, aiming at the analysis of the whole process of a single bridge. \\
\hline [20] & $\begin{array}{l}\text { The research focuses on the possibility of, problems with and knowledge gap related to the use of product platforms } \\
\text { in the construction environment, and their popularization needs to be further studied. }\end{array}$ \\
\hline [21] & $\begin{array}{l}\text { The research is carried out in Chile. In order to transfer the results to any other country, the diversity of } \\
\text { environments and climates in other countries must be taken into account. }\end{array}$ \\
\hline [22] & $\begin{array}{l}\text { The diversity of construction projects and the uncertainty of information sources have an impact on productivity, } \\
\text { and the framework has limitations. }\end{array}$ \\
\hline [23] & The research focuses on the theoretical derivation and analysis. \\
\hline [24] & The results of the effect on the success of the project cannot be applied or confirmed. \\
\hline [25] & $\begin{array}{l}\text { We studied samples from Europe, North America, Australia, and other countries, and determined that the } \\
\text { complexity of the project is the main criterion for success. There is a lack of further research on whether the project } \\
\text { is consistent with international projects. }\end{array}$ \\
\hline [26] & $\begin{array}{l}\text { The study dealt with the implementation of green building from the planning phase, without an in-depth study of } \\
\text { the entire life cycle. Whether the application of the concept is in line with the implementation of international } \\
\text { infrastructure remains to be further studied. }\end{array}$ \\
\hline [27] & $\begin{array}{l}\text { Whether the research field can be expanded, the evaluation and optimization criteria and the selection of the best } \\
\text { scheme need to be improved. }\end{array}$ \\
\hline [28] & $\begin{array}{l}\text { This paper studies the reasons for promoting the development of green building by modeling. The specific } \\
\text { construction environment, specific regional model, specific conditions, and expansion research require innovative } \\
\text { analysis. }\end{array}$ \\
\hline [29] & $\begin{array}{l}\text { The model is mainly used for the design and planning of a supply chain operating in the Gao Bei region. Whether it } \\
\text { is fully applicable to supply chains operating in other regions needs to be studied. }\end{array}$ \\
\hline [30] & $\begin{array}{l}\text { In the case of limited uncertainty, a case study is proposed on how to maintain environmental and social benefits. It } \\
\text { requires fine research and promotion. }\end{array}$ \\
\hline [31] & $\begin{array}{l}\text { There are many factors considered in the research process, and the depth needs to be specified. Focusing on the } \\
\text { impact factors would strengthen the depth of the analysis. }\end{array}$ \\
\hline [32] & $\begin{array}{l}\text { In the early stages of the application of fuzzy analysis, this paper considers the explanation of the basic concepts of } \\
\text { fuzzy mathematics, and in the later stages, it considers the problem of distinguishing membership degrees for } \\
\text { important influencing factors. }\end{array}$ \\
\hline [33] & The lack of application of research results, analysis methods and factors of the problem. \\
\hline
\end{tabular}




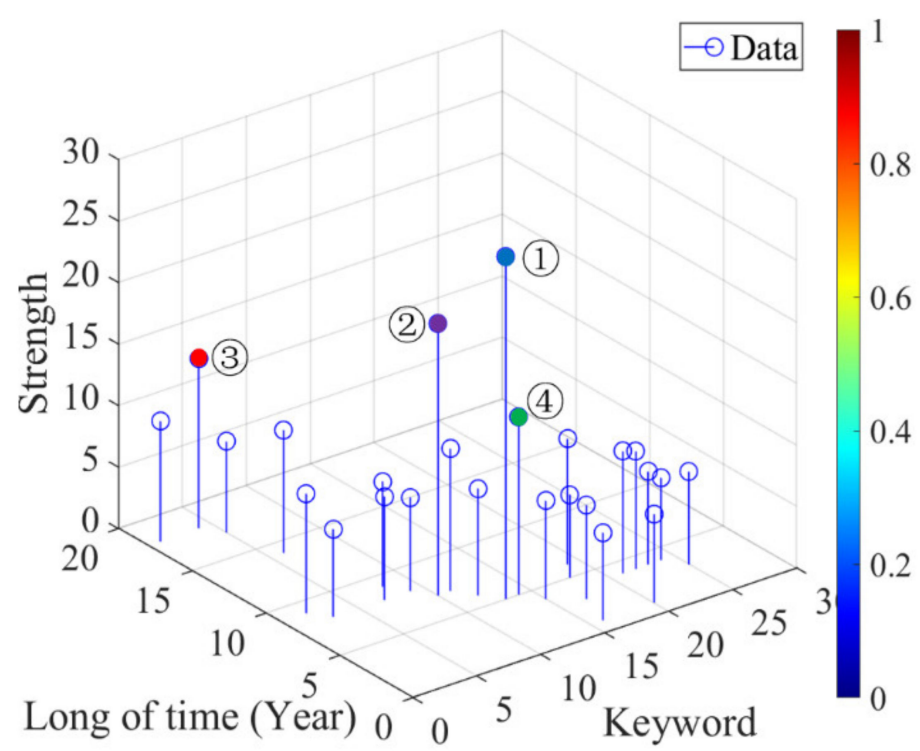

(a)

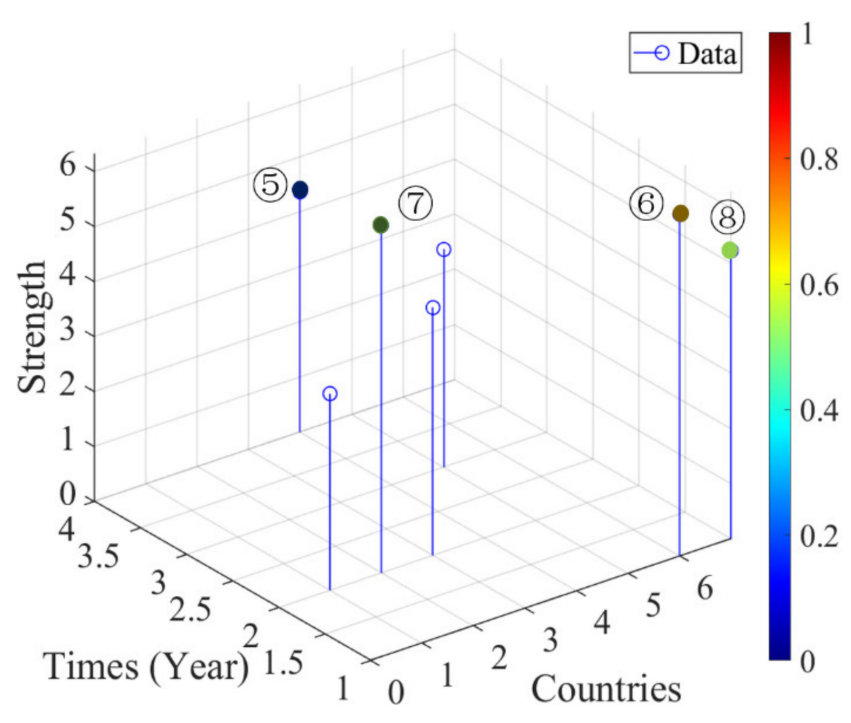

(b)

Figure 3. Literature clustering network analysis: (a) Keyword clustering network map analysis; (b) Institution and country clustering network map analysis (the node connection graph that highlights the main keywords, the higher the centrality and the higher the value of the node).

As a result of the above analysis, there is a need to intensify research in project management and environmental impact assessment analysis. It should not be limited to some regions and countries. Detailed findings of the evaluation of representative articles can be found in Table 1 from 2012 to the present.

Unfortunately, no software and system can determine the project management of construction works. The reason is that construction works are a dynamic layout and planning process, with dynamic characteristics such as spatial planning, spatio-temporal dispatching, flow pitch time control, integration of scattered industries, and on-site risk control. Staff uncertainty intensifies the complexity of the project entity [34].

Through research and analysis of scientific research results in the direction of project management in the past 52 years, it is found that the environmental impact is not used as the assessment standard to improve the project management. It has a key guiding role in reducing the pollution of the construction industry to the global environment. In the published research results, the comprehensive environmental, economic, and social factors are not added to the framework of the project management theory system, which is missing in terms of sustainability evaluation; a new comprehensive evaluation framework sys-tem for sustainable development needs to be established.

\subsection{Current International System Assessment}

Provided enough research results on international project management (Figures 1 and 2), focusing on cost, schedule, quality, and safety in the setting of project management framework. Different organizations have small differences in the management framework, but the overall assessment direction is the same. There is a lack of using environmental impact as one of the project management assessment criteria. There is no environment-related index evaluation standard, particularly in the index for measuring project success (using the Delphi method).

Figure 4 lists the main current international project management and evaluation systems, which are used as the reference evaluation system for this research. The aim of this work is how to effectively reduce the environmental impact of the construction phase through project management as well as how to evaluate and optimize the solution. The first premise is to ensure the safety, quality, progress, specification, and other conditions of the project, to minimize environmental pollution and achieve sustainable development. 


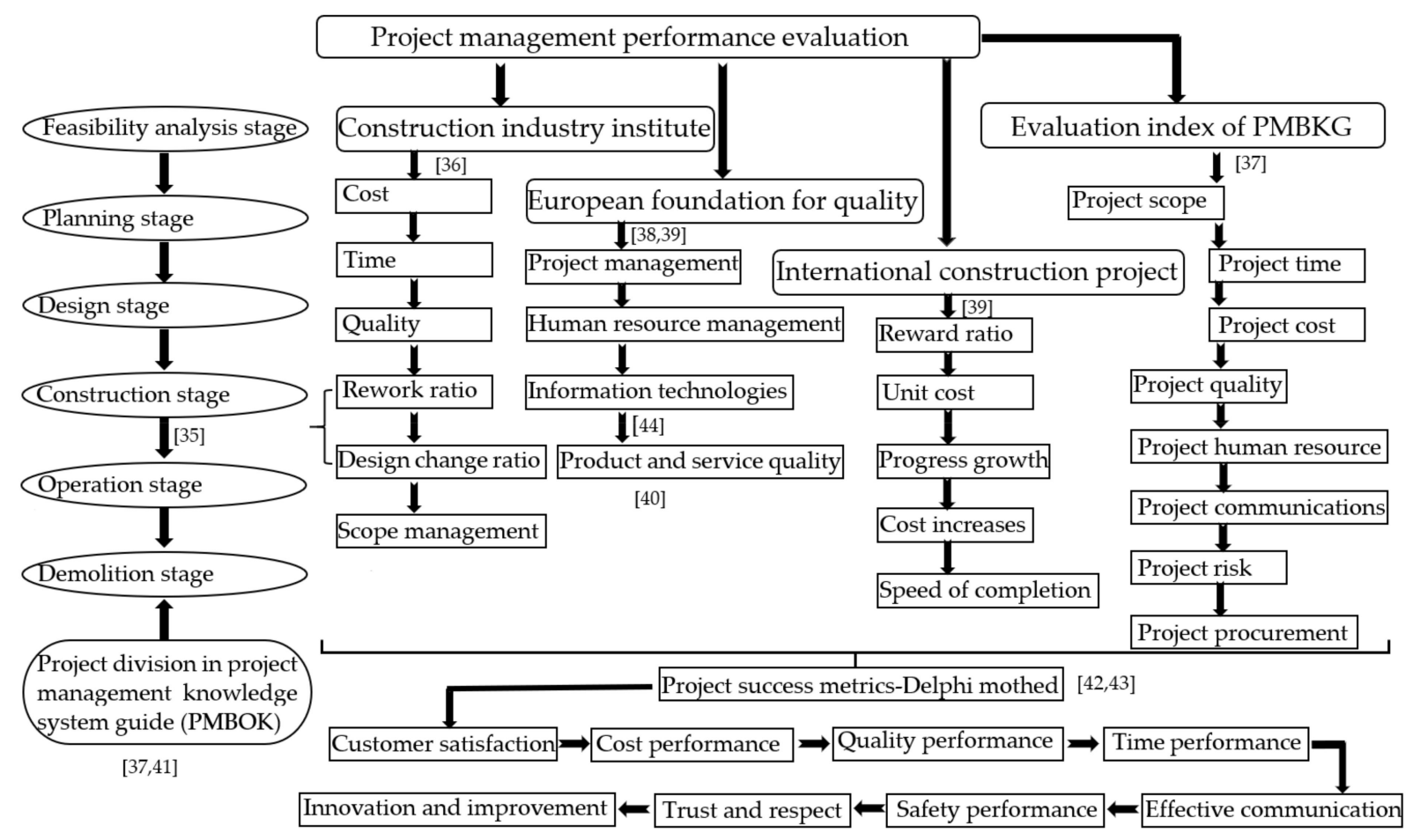

Figure 4. Four types of project management evaluation system and international project success index statistics [35-42].

In this paper, a large and complex bridge case was selected to analyze and verify the robustness of the proposal. In the research process, the concept of sustainable development management was applied, and sustainability was optimized by analyzing data from the four designed project management models.

The purpose is to establish a framework for the evaluation of the sustainable development project management that is suitable for global development; to address current gaps in research in this field, and to reform the management system for the green construction industry.

The innovation of the work lies in the establishment of a new theoretical system after taking into account the interference of various influencing factors of the environment, economy, society, and project management framework, and applying the established framework to compare and analyze the results of case project management environment and cost evaluation. The project management system standard has been optimized, upgraded and innovated, and a comprehensive evaluation framework system for sustainable development has been realized.

\section{Methodology and Modeling}

Zhou et al. [5] investigated the environmental, economic, and social impacts of six bridges in five provinces of China, and accurately determined the evaluation framework and theoretical model $[43,44]$.

According to the analysis results of Table 1, Figures 1 and 2 the bridge project management framework is established.

Definition 1. Assumes $X_{1}, X_{2}, \cdots \cdots X_{n}$ is a discrete sequence of project management variables, abbreviated as $\left\{X_{n}\right\}, X_{n}$ All possible values of $N$ are called $\left\{X_{n}\right\}$ space, record as $E=$ $\left\{x_{1}, x_{2}, \cdots \cdots x_{n}\right\}$. For any $n>0$ and $x_{i_{1}}, x_{i_{1 n}}, x_{i_{1 n+1}} \in E$, and $p\left(X_{n+1}=x_{i_{n+1}}\right)$ $\left.\mid X_{1}=x_{i_{1}}, X_{2}=x_{i_{2}}, \ldots \ldots X_{n}=x_{i_{n}}\right)$. 
Definition 2. Project management is affected by multiple variables of $p\left(X_{n+1}=x_{j}\right)$ impact, denoted as $p_{i j}(k)$ which is called $p_{i j}(k)\left(x_{i}, x_{j} \in E\right)$ The random matrix with $p_{i j}(k)\left(x_{i}, x_{j} \in E\right)$ as a variable is denoted as $A(k)$.

$$
\mathrm{A}(\mathrm{k})=\left[\begin{array}{cccc}
\mathrm{p}_{11}(\mathrm{k}) & \mathrm{p}_{12}(\mathrm{k}) & \cdots & \mathrm{p}_{1 \mathrm{j}}(\mathrm{k}) \\
\mathrm{p}_{21}(\mathrm{k}) & \mathrm{p}_{22}(\mathrm{k}) & \cdots & \mathrm{p}_{2 \mathrm{j}}(\mathrm{k}) \\
\vdots & \vdots & \vdots & \vdots \\
\mathrm{p}_{\mathrm{i} 1}(\mathrm{k}) & \mathrm{p}_{\mathrm{i} 2}(\mathrm{k}) & \cdots & \mathrm{p}_{\mathrm{ij}}(\mathrm{k})
\end{array}\right]
$$

The results of the research by Zhou et al. [5] divide the project into five stages: design, material, construction, maintenance, and recycling. Dikmen et al. [45] studied the relationship between the key factors of project management, and built models using the Bayesian belief network, a network such that $A_{1} \rightarrow A_{2} \cdots \rightarrow A_{1}$ thereby creating a cycle. For a Bayesian network specified over $A=A_{1}, \cdots A_{n}$, the unique joint probability distribution $\mathrm{P}(\mathrm{A})$ representing the product of all conditional probability tables is given in Equation (1):

$$
\mathrm{P}(\mathrm{A})=\prod_{\mathrm{i}=1}^{\mathrm{n}} \mathrm{P}\left(\mathrm{A}_{\mathrm{i}} \mid \mathrm{Pa}\left(\mathrm{A}_{\mathrm{i}}\right)\right)
$$

where $\mathrm{P}(\mathrm{A})$ are the parents of $\mathrm{A}_{\mathrm{i}}$. According to the complexity of the influencing factors in Figure 4 , the matching degree between features and project management is optimized, and the concept of membership degree is introduced into the influence degree of project management.

Each evaluation standard of project management is set as element $\mathrm{u}_{1}$. In the range of closed interval [0,1], a corresponding numerical index is given according to the degree of influence to express $u_{1}$ The subordinate degree of $u_{1}$ to project management $p$, which is expressed by $\mu_{p}$, is called element $u_{1}$ to $P$, and $0 \leq P \leq 1$ [46].

$$
\mathrm{P}(\mathrm{u})=\left[\begin{array}{cc}
\mathrm{p}_{\mu_{\mathrm{p} 1}} & 0 \leq \mu_{\mathrm{p} 1} \leq 1 \\
\mathrm{p}_{\mu_{\mathrm{p} 2}} & 0 \leq \mu_{\mathrm{p} 2} \leq 1 \\
\vdots & \vdots \\
\vdots & \vdots \\
\mathrm{p}_{\mu_{\mathrm{pn}}} & 0 \leq \mu_{\mathrm{pn}} \leq 1
\end{array}\right]
$$

From the Expressions (1), (2) and (3):

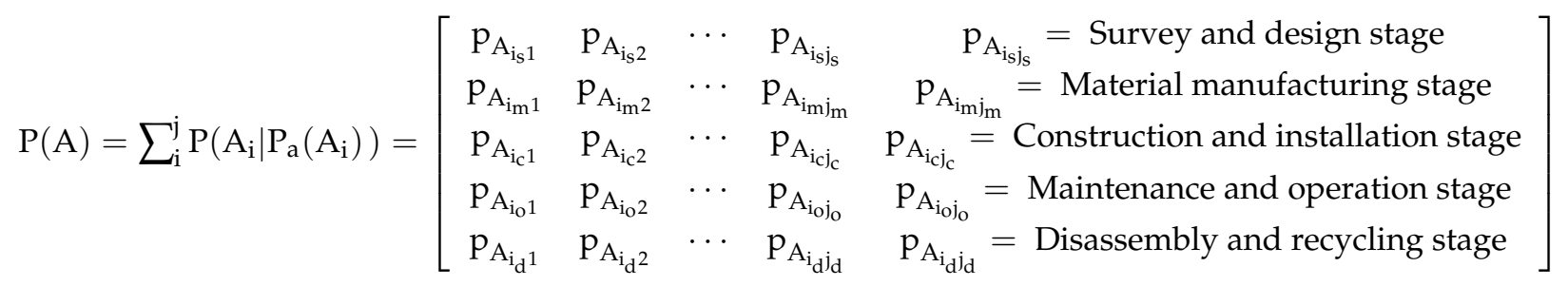

$\mathrm{P}(\mathrm{A})=$ Project management impact (Environmental unit: kg; Economic unit: Chinese Yuan: (CNY); Social impact unit: Med risk hours).

$$
\begin{gathered}
\mathrm{p}_{\mathrm{A}_{\mathrm{i} j_{\mathrm{s}}}}=\left[\begin{array}{ccccc}
\mathrm{p}_{\mathrm{i} 1}\left(\mathrm{E}_{\mathrm{s}}\right) & \mathrm{p}_{\mathrm{i} 2}\left(\mathrm{E}_{\mathrm{s}}\right) & \cdots & \mathrm{p}_{\mathrm{ij}}\left(\mathrm{E}_{\mathrm{s}}\right) & \mathrm{p}_{\mathrm{ij}}\left(\mathrm{E}_{\mathrm{s}}\right)=\text { Environmental impact } \\
\mathrm{p}_{\mathrm{i} 1}\left(\mathrm{EC}_{\mathrm{s}}\right) & \mathrm{p}_{\mathrm{i} 2}\left(E C_{\mathrm{s}}\right) & \cdots & \mathrm{p}_{\mathrm{ij}}\left(\mathrm{EC}_{\mathrm{s}}\right) & \mathrm{p}_{\mathrm{ij}}\left(\mathrm{EC}_{\mathrm{s}}\right)=\text { Economic impact } \\
\mathrm{p}_{\mathrm{i} 1}\left(\mathrm{~S}_{\mathrm{s}}\right) & \mathrm{p}_{\mathrm{i} 2}\left(\mathrm{~S}_{\mathrm{s}}\right) & \cdots & \mathrm{p}_{\mathrm{ij}}\left(\mathrm{S}_{\mathrm{s}}\right) & \mathrm{p}_{\mathrm{ij}}\left(\mathrm{S}_{\mathrm{s}}\right)=\text { Social impact }
\end{array}\right] \\
\mathrm{p}_{\mathrm{A}_{\mathrm{im} j_{\mathrm{m}}}}=\left[\begin{array}{ccccc}
\mathrm{p}_{\mathrm{i} 1}\left(\mathrm{E}_{\mathrm{m}}\right) & \mathrm{p}_{\mathrm{i} 2}\left(\mathrm{E}_{\mathrm{m}}\right) & \cdots & \mathrm{p}_{\mathrm{ij}}\left(\mathrm{E}_{\mathrm{m}}\right) & \mathrm{p}_{\mathrm{ij}}\left(\mathrm{E}_{\mathrm{m}}\right)=\text { Environmental impact } \\
\mathrm{p}_{\mathrm{i} 1}\left(E C_{\mathrm{m}}\right) & \mathrm{p}_{\mathrm{i} 2}\left(E C_{\mathrm{m}}\right) & \cdots & \mathrm{p}_{\mathrm{ij}}\left(E C_{\mathrm{m}}\right) & \mathrm{p}_{\mathrm{ij}}\left(E C_{\mathrm{m}}\right)=\text { Economic impact } \\
\mathrm{p}_{\mathrm{i} 1}\left(\mathrm{~S}_{\mathrm{m}}\right) & \mathrm{p}_{\mathrm{i} 2}\left(\mathrm{~S}_{\mathrm{m}}\right) & \cdots & \mathrm{p}_{\mathrm{ij}}\left(\mathrm{S}_{\mathrm{m}}\right) & \mathrm{p}_{\mathrm{ij}}\left(\mathrm{S}_{\mathrm{m}}\right)=\text { Social impact }
\end{array}\right]
\end{gathered}
$$




$$
\begin{aligned}
& \mathrm{p}_{\mathrm{A}_{\mathrm{i} \mathrm{i}_{\mathrm{c}}}}=\left[\begin{array}{ccccc}
\mathrm{p}_{\mathrm{i} 1}\left(\mathrm{E}_{\mathrm{c}}\right) & \mathrm{p}_{\mathrm{i} 2}\left(\mathrm{E}_{\mathrm{c}}\right) & \cdots & \mathrm{p}_{\mathrm{ij}}\left(\mathrm{E}_{\mathrm{c}}\right) & \mathrm{p}_{\mathrm{ij}}\left(\mathrm{E}_{\mathrm{c}}\right)=\text { Environmental impact } \\
\mathrm{p}_{\mathrm{i} 1}\left(\mathrm{EC}_{\mathrm{c}}\right) & \mathrm{p}_{\mathrm{i} 2}\left(\mathrm{EC}_{\mathrm{c}}\right) & \cdots & \mathrm{p}_{\mathrm{ij}}\left(\mathrm{EC}_{\mathrm{c}}\right) & \mathrm{p}_{\mathrm{ij}}\left(\mathrm{EC}_{\mathrm{c}}\right)=\text { Economic impact } \\
\mathrm{p}_{\mathrm{i} 1}\left(\mathrm{~S}_{\mathrm{c}}\right) & \mathrm{p}_{\mathrm{i} 2}\left(\mathrm{~S}_{\mathrm{c}}\right) & \cdots & \mathrm{p}_{\mathrm{ij}}\left(\mathrm{S}_{\mathrm{c}}\right) & \mathrm{p}_{\mathrm{ij}}\left(\mathrm{S}_{\mathrm{c}}\right)=\text { Social impact }
\end{array}\right] \\
& \mathrm{p}_{\mathrm{A}_{\mathrm{i}_{\mathrm{oj}}}}=\left[\begin{array}{ccccc}
\mathrm{p}_{\mathrm{i} 1}\left(\mathrm{E}_{\mathrm{o}}\right) & \mathrm{p}_{\mathrm{i} 2}\left(\mathrm{E}_{\mathrm{o}}\right) & \cdots & \mathrm{p}_{\mathrm{ij}}\left(\mathrm{E}_{\mathrm{o}}\right) & \mathrm{p}_{\mathrm{ij}}\left(\mathrm{E}_{\mathrm{o}}\right)=\text { Environmental impact } \\
\mathrm{p}_{\mathrm{i} 1}\left(E C_{\mathrm{o}}\right) & \mathrm{p}_{\mathrm{i} 2}\left(E C_{\mathrm{o}}\right) & \cdots & \mathrm{p}_{\mathrm{ij}}\left(E C_{\mathrm{o}}\right) & \mathrm{p}_{\mathrm{ij}}\left(E C_{\mathrm{o}}\right)=\text { Economic impact } \\
\mathrm{p}_{\mathrm{i} 1}\left(\mathrm{~S}_{\mathrm{o}}\right) & \mathrm{p}_{\mathrm{i} 2}\left(\mathrm{~S}_{\mathrm{o}}\right) & \cdots & \mathrm{p}_{\mathrm{ij}}\left(\mathrm{S}_{\mathrm{o}}\right) & \mathrm{p}_{\mathrm{ij}}\left(\mathrm{S}_{\mathrm{o}}\right)=\text { Social impact }
\end{array}\right] \\
& \mathrm{p}_{\mathrm{A}_{\mathrm{i} d \mathrm{~d}}}=\left[\begin{array}{ccccc}
\mathrm{p}_{\mathrm{i} 1}\left(\mathrm{E}_{\mathrm{d}}\right) & \mathrm{p}_{\mathrm{i} 2}\left(\mathrm{E}_{\mathrm{d}}\right) & \cdots & \mathrm{p}_{\mathrm{ij}}\left(\mathrm{E}_{\mathrm{d}}\right) & \mathrm{p}_{\mathrm{ij}}\left(\mathrm{E}_{\mathrm{d}}\right)=\text { Environmental impact } \\
\mathrm{p}_{\mathrm{i} 1}\left(\mathrm{EC}_{\mathrm{d}}\right) & \mathrm{p}_{\mathrm{i} 2}\left(\mathrm{EC}_{\mathrm{d}}\right) & \cdots & \mathrm{p}_{\mathrm{ij}}\left(\mathrm{EC}_{\mathrm{d}}\right) & \mathrm{p}_{\mathrm{ij}}\left(\mathrm{EC}_{\mathrm{d}}\right)=\text { Economic impact } \\
\mathrm{p}_{\mathrm{i} 1}\left(\mathrm{~S}_{\mathrm{d}}\right) & \mathrm{p}_{\mathrm{i} 2}\left(\mathrm{~S}_{\mathrm{d}}\right) & \cdots & \mathrm{p}_{\mathrm{ij}}\left(\mathrm{S}_{\mathrm{d}}\right) & \mathrm{p}_{\mathrm{ij}}\left(\mathrm{S}_{\mathrm{d}}\right)=\text { Social impact }
\end{array}\right]
\end{aligned}
$$

$\mathrm{s}=$ Design stage $\mathrm{m}=$ Material stage $\mathrm{c}=$ Construction stage $\mathrm{o}=$ Maintenance stage; $\mathrm{d}=$ Recycling stage $; \mathrm{E}=$ Environmental impact $; \mathrm{S}=$ Social impact $; \mathrm{EC}=$ Economic impact.

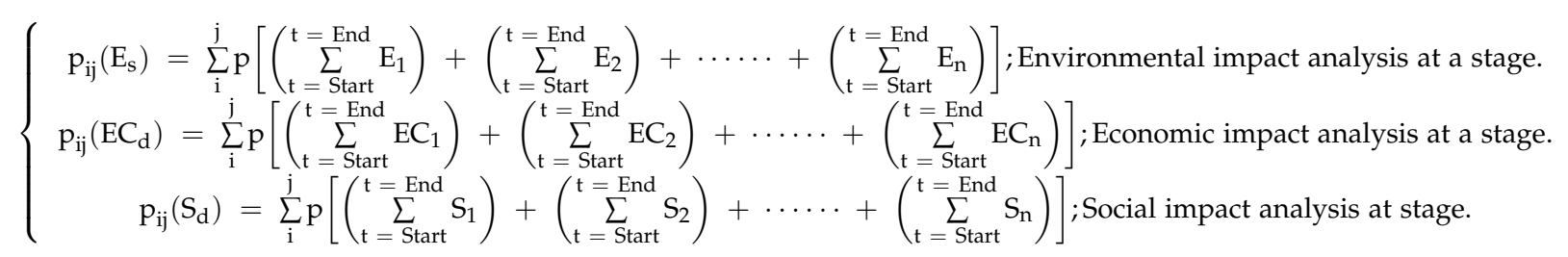

Definition 3. Formula (11) is the final framework formula of different impact factors in each stage of project management. Formulas (3) and (10) and Figure 4 can be used to deduce the comprehensive judgment standard model.

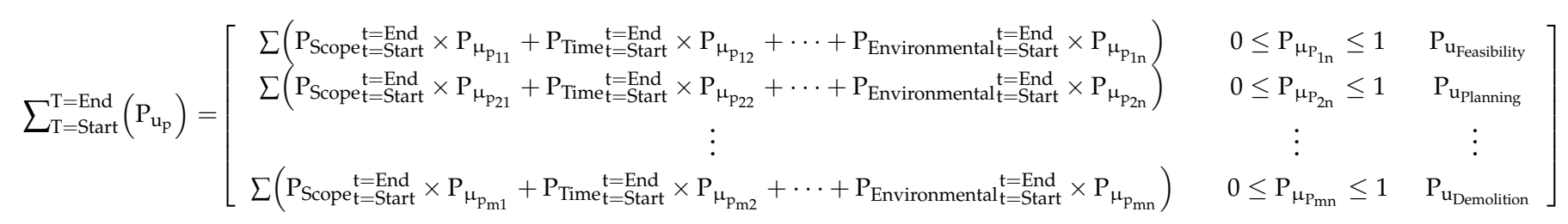

$\mathrm{P}_{\mathrm{T}}^{\mathrm{T}}=$ = End $\left(\sum_{1}^{\mathrm{n}} \mathrm{p}_{\mathrm{u}_{\mathrm{p}}}\right)=$ Comprehensive evaluation standard of project management;

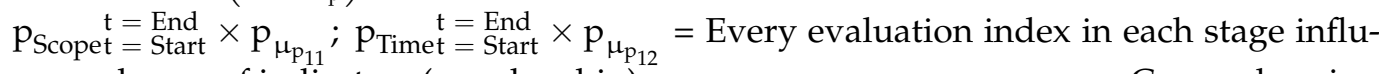
ences degree of indicators (membership); $\mathrm{p}_{\mathrm{u}_{\text {Feasibility }}} ; \mathrm{p}_{\mathrm{u}_{\text {Planing }}} ; \mathrm{p}_{\mathrm{u}_{\text {Demolition }}}=$ Comprehensive evaluation value of each stage; Expression (10) is the final mathematical modeling conclusion of project management evaluation theory.

\subsection{Construction Project Management}

The construction industry is one of the pillar industries of the national economy, but it has many disadvantages such as excessive energy consumption and a low level of mechanization. Countries all over the world have put forward production models of construction industrialization to improve the existing problems and promote the sustainable development of environmental, economic, and social benefits [47-50]. As the industrial technologies develop and expand, construction project management is integrating building information modeling, augmented reality, virtual reality, the Internet of Things, and block chain technology to achieve scientific management [51]. Construction production is supply chain management based on a project, featuring a more complex and dynamic production process, and involving more participating members, for example designers, supervisors, general construction parties, subcontractors, professional contractors, materials suppliers, and labor staff service companies [52].

Figure 5 shows the detailed process of project management and the key points of management control in the construction phase: the element control of the main nodes is the key to the success or failure of the project. 


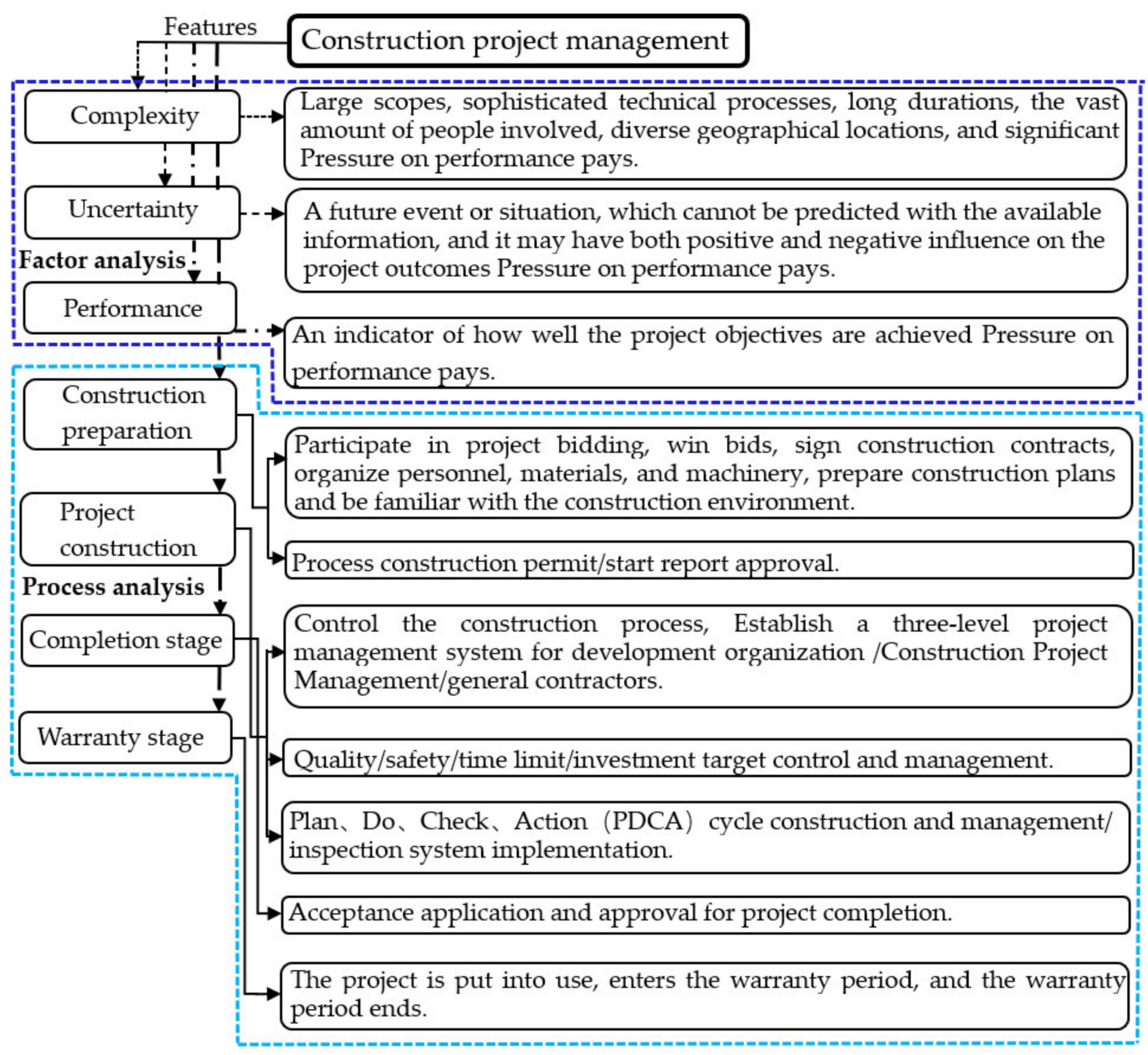

Figure 5. Construction project management factors and process analysis diagram [53-55].

\subsection{Environmental Impact during the Construction Phase}

This study mainly analyses the environmental impact contribution in the construction stage of a bridge to design the project management, reduce the construction costs and reduce environmental pollution. Zhou et al. [56] studied the environmental impact contribution of a cable-stayed bridge throughout its lifecycle in detail, and accurately defined the environmental impact of the construction stage as within the range of $14.7-34.1 \%$ of the total contribution. The results show that an effective project management model is essential.

In this study, the material manufacturing and construction stages were collectively referred to as the project construction stage.

Environmental impact of raw materials:

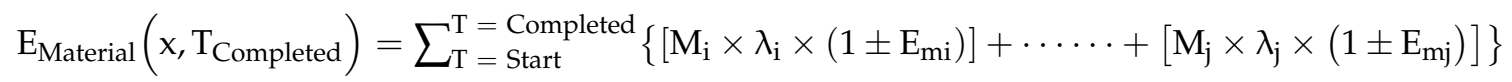

$\mathrm{E}_{\text {Material }}\left(\mathrm{x}, \mathrm{T}_{\text {Completed }}\right)=$ Impact contribution of materials in the project construction stage $(\mathrm{kg}) ; \mathrm{M}_{\mathrm{i}}, \mathrm{M}_{\mathrm{j}}=$ Total quantity of materials $\mathrm{i}, \mathrm{j} ; \lambda_{\mathrm{i}}, \lambda_{\mathrm{j}}=$ Emission coefficient of materials $\mathrm{i}, \mathrm{j}(\mathrm{kg}) ; \mathrm{E}_{\mathrm{mi}}, \mathrm{E}_{\mathrm{mj}}=$ Loss coefficient of materials $\mathrm{i}, \mathrm{j}(\%)$.

Environmental impact of transport vehicles:

$$
T_{\text {Vehicle }}\left(x, T_{\text {Completed }}\right)=\sum_{T=\text { Start }}^{T=\text { Completed }}\left\{\begin{array}{c}
{\left[G_{k} \times D_{k} \times \lambda_{n} \times\left(1 \pm E_{n k}\right)\right]+\cdots \cdots+\left[G_{l} \times D_{1} \times \lambda_{m} \times\left(1 \pm E_{m l}\right)\right] \text { Real vehicles One way }} \\
{\left[G_{s} \times D_{k} \times \lambda_{n} \times\left(1 \pm E_{n s}\right)\right]+\cdots \cdots+\left[G_{q} \times D_{1} \times \lambda_{m} \times\left(1 \pm E_{m q}\right)\right] \text { Empty vehicles }_{\text {One way }}}
\end{array}\right\}
$$


$\mathrm{T}_{\text {Vehicle }}\left(\mathrm{x}, \mathrm{T}_{\text {Completed }}\right)=$ Impact contribution of transport vehicle $(\mathrm{kg}) ; \mathrm{G}_{\mathrm{k}}, \mathrm{G}_{\mathrm{l}}=$ Fuel consumption of truck under load $(\mathrm{kg} / 100 \mathrm{~km}) ; \mathrm{D}_{\mathrm{k}}, \mathrm{D}_{1}=$ Shipment distance of single trip; $\lambda_{n}, \lambda_{\mathrm{m}}=$ Emission coefficient of fuel $\mathrm{n}, \mathrm{m}(\mathrm{kg}) ; \mathrm{E}_{\mathrm{nk}}, \mathrm{E}_{\mathrm{ml}}=$ Loss coefficient of fuel $\mathrm{n}, \mathrm{m}$ $\left(\%,[57] ; \mathrm{G}_{\mathrm{s}}, \mathrm{G}_{\mathrm{q}}=\right.$ Fuel consumption of truck under no-load $(\mathrm{kg} / 100 \mathrm{~km})$.

Environmental impact of construction equipment: $\mathrm{E}_{\text {Mechanical }}\left(\mathrm{x}, \mathrm{T}_{\text {Completed }}\right)=\sum_{\mathrm{i}}^{1}\left\{\left(\mathrm{E}_{\mathrm{mj}} \times \mathrm{T}_{\mathrm{j}} \times \lambda_{\mathrm{j}}\right)+\cdots \cdots+\left[\mathrm{E}_{\mathrm{m} 1} \times\left(1+\mathrm{E}_{1}\right) \times \mathrm{T}_{1} \times \lambda_{1}\right]\right\}$.

$E_{\text {Mechanical }}\left(x, T_{\text {Completed }}\right)=$ Impact contribution of equipment $(\mathrm{kg}) ; E_{m j}, E_{m l}=$ Fuel consumption and power consumption of equipment $\mathrm{j}, l ; T_{j}, T_{l}=$ Effective working hours of equipment $\mathrm{j}, l$ (Hour); $E_{l}=$ Power consumption (\%, equipment impedance value [58]; $\lambda_{j}, \lambda_{l}=$ Emission coefficient of fuel and electric energy of equipment $\mathrm{j}, l(\mathrm{~kg})$.

Environmental impact of wastes and sewage discharged by staff:

$$
\mathrm{E}_{\text {Staff }}\left(\mathrm{x}, \mathrm{T}_{\text {Completed }}\right)=\sum_{\mathrm{T}=\text { Start }}^{\mathrm{T}=\text { Completed }}\left(\mathrm{P}_{\mathrm{a}} \times \mathrm{T}_{\mathrm{m}} \times \mathrm{T}_{\mathrm{n}} \times \lambda_{\mathrm{p}}+\mathrm{S}_{\mathrm{m}} \times \mathrm{P}_{\mathrm{a}} \times \lambda_{\mathrm{x}} \times \mathrm{T}_{\mathrm{n}}\right)
$$

$\mathrm{E}_{\text {Staff }}\left(\mathrm{x}, \mathrm{T}_{\text {Completed }}\right)=$ Impact contribution of wastes and sewage discharged by staff $(\mathrm{kg}) ; \mathrm{P}_{\mathrm{a}}=$ Total number of staff (Persons); $\mathrm{T}_{\mathrm{m}}=$ Domestic waste $\left(\mathrm{kg} /\right.$ day); $\mathrm{T}_{\mathrm{n}}=$ Time in the post of staff (day); $\lambda_{\mathrm{p}}=$ Emission coefficient of domestic waste $(\mathrm{kg})[59] ; \mathrm{S}_{\mathrm{m}}=$ Sewage generated by staff $\left(\mathrm{kg} /\right.$ day); $\lambda_{\mathrm{x}}=$ Emission coefficient of sewage $(\mathrm{kg})$ [60].

Environmental impact of energy consumption:

$$
\mathrm{E}_{\text {Energy }}\left(\mathrm{x}, \mathrm{T}_{\text {Completed }}\right)=\sum_{\mathrm{T}=\text { Start }}^{\mathrm{T}=\text { Completed }}\left\{\begin{array}{cc}
{\left[\mathrm{T}_{\mathrm{i}} \times \lambda_{\mathrm{p}} \times\left(1+\mathrm{E}_{1}\right)\right]} & \text { Staff }_{\text {Electricity consumption }} \\
{\left[\mathrm{T}_{1} \times \lambda_{\mathrm{q}} \times\left(1+\mathrm{E}_{\mathrm{W}}\right)\right]} & \text { Staff }_{\text {Water consumption }} \\
\mathrm{G}_{\mathrm{m}} \times \lambda_{\mathrm{n}} \times \mathrm{T}_{\mathrm{m}} \times\left(1+\mathrm{L}_{\mathrm{n}}\right) & \text { Generator }_{\text {Fuel consumption }} \\
\text { Energy consumption } & \text { Special case }_{\text {Other energy }}
\end{array}\right\}
$$

$\mathrm{E}_{\text {Energy }}\left(\mathrm{x}, \mathrm{T}_{\text {Completed }}\right)=$ Impact contribution of energy in the construction stage $(\mathrm{kg})$; $\mathrm{T}_{\mathrm{i}}, \mathrm{T}_{1}=$ Daily energy consumption and water consumption of staff (kW/day, kg/day) [61]; $\lambda_{\mathrm{p}}, \lambda_{\mathrm{q}}=$ Emission coefficient of electricity and water $(\mathrm{kg} / \mathrm{kW}, \mathrm{kg}) ; \mathrm{E}_{\mathrm{w}}=$ Loss coefficient of water (\%); $\mathrm{G}_{\mathrm{m}}=$ Fuel consumption of engine at the time of power outage and field operation (kg/hour); $\lambda_{\mathrm{n}}=$ Emission coefficient of fuel $\mathrm{n}(\mathrm{kg}) ; \mathrm{T}_{\mathrm{m}}=$ Working time of power generation equipment (hour); $\mathrm{L}_{\mathrm{n}}=$ Fuel consumption during working time of power generation equipment $(\mathrm{kg} /$ hour $)$.

\subsection{Project Construction Economic Cost}

Frangopol et al. [62]define the lifecycle cost (LCC) of a bridge structure as the total cost incurred during the service life of the structure, including the cost of design, construction, inspection, maintenance, and repair, and determine the modelling equation as follows:

$$
\mathrm{LCC}=\mathrm{C}_{\mathrm{r}}+\mathrm{C}_{\mathrm{PM}}+\mathrm{C}_{\mathrm{INS}}+\mathrm{C}_{\mathrm{REP}}+\mathrm{C}_{\mathrm{F}}+\mathrm{C}_{\mathrm{D}}
$$

where $C_{r}=$ The initial cost $(\mathrm{CNY}) ; \mathrm{C}_{\mathrm{PM}}=$ The maintenance $\operatorname{cost}(\mathrm{CNY}) ; \mathrm{C}_{\mathrm{INS}}=$ The inspections $\operatorname{cost}(\mathrm{CNY}) ; \mathrm{C}_{\mathrm{REP}}=$ The repair $\operatorname{cost}(\mathrm{CNY}) ; \mathrm{C}_{\mathrm{F}}=$ The failure cost, and $\mathrm{C}_{\mathrm{D}}=$ The demolition cost (CNY).

Project construction costs include direct and indirect costs. Direct costs include project materials, transportation, labor, and equipment costs. Indirect costs include environmental and social losses [63]. According to the environmental protection law of the new era, Wang et al. [64] Express LCC as:

$$
\mathrm{LCC}=\mathrm{C}_{\mathrm{DIR}}+\mathrm{C}_{\mathrm{E}}+\mathrm{C}_{\mathrm{S}}
$$

where $C_{D I R}=$ The direct costs $(C N Y) ; C_{S}=$ The overhead costs $(C N Y)$, and $C_{E}=$ The environmental costs (CNY). 
The research case is a cable-stayed bridge in China. The economic cost is analyzed according to the "China Transportation Industry Standard", "JTG 3830-2018 Highway Estimate Standard" and "JTG/T 3831-2018 Specification" [65]. The calculation of project construction costs (as shown in Table 2) is as follows:

$$
\mathrm{C}_{\text {Build }}\left(\overline{\mathrm{x}}, \mathrm{T}_{\text {End }}\right)=\sum_{\mathrm{t}=\text { Start }}^{\mathrm{T}_{\text {Warranty termination }}}\left\{\frac{\mathrm{C}_{\text {Direct cost }}+\mathrm{C}_{\text {Extra charge }}+\left[\left(\mathrm{C}_{\text {Direct cost }}+\mathrm{C}_{\text {Extra charge }}\right) * \mathrm{C}_{\text {Profit }}\right]}{(1+\mathrm{r})^{\mathrm{t}}}\right\}\left[1 \pm\left(\mathrm{R}_{\mathrm{t}}\right)\right]
$$

Table 2. Budget process of project. Unit: CNY.

\begin{tabular}{|c|c|c|c|}
\hline Number & Cost Name & Tax Rate & Calculation Method \\
\hline 1 & Direct project cost & Quote the fixed tax rate. & According to "JTG 3830-2018" budget \\
\hline 2 & insurance & $3.00 \%$ & $1 \times 2$ \\
\hline 3 & Environmental protection fee & $1,500,000$ & Fixed costs \\
\hline 4 & Safety production fee & $1.50 \%$ & $1 \times 4$ \\
\hline 5 & Management information fee & 200,000 & Fixed costs \\
\hline 6 & $\begin{array}{l}\text { Temporary road construction, } \\
\text { maintenance, and demolition }\end{array}$ & $0.16 \%$ & $1 \times 8$ \\
\hline 7 & $\begin{array}{l}\text { Temporary land occupation, } \\
\text { occupying the river }\end{array}$ & $0.25 \%$ & $1 \times 9$ \\
\hline 8 & $\begin{array}{l}\text { Temporary alms for project } \\
\text { construction }\end{array}$ & $0.24 \%$ & $1 \times 8$ \\
\hline 9 & $\begin{array}{c}\text { Standard chemical site } \\
\text { construction) }\end{array}$ & $0.42 \%$ & $1 \times 9$ \\
\hline 10 & Provisional amount & $5.00 \%$ & $(1+2+3+4+5+6=7+8+9) \times 10$ \\
\hline 11 & Project construction cost & & $11=1+\ldots \ldots \ldots \ldots+10$ \\
\hline
\end{tabular}

Here, $\mathrm{C}_{\text {Build }}\left(\overline{\mathrm{x}}, \mathrm{T}_{\text {End }}\right)=\mathrm{LCC}$ assessment cost in the construction stage $(\mathrm{CNY}) ; \mathrm{C}_{\text {Direct cost }}$ $=$ Direct cost $(\mathrm{CNY}) ; \mathrm{C}_{\text {Extra charge }}=$ Indirect cost $(\mathrm{CNY}) ; \mathrm{C}_{\text {Profit }}=$ Construction profit $(\mathrm{CNY})$; $\mathrm{R}_{\mathrm{t}}=$ National tax rate $(\%) ; \mathrm{r}=$ Discount rate $(\%)$. The costs incurred for $\mathrm{C}_{\mathrm{E}}$ and $\mathrm{C}_{\mathrm{S}}$ are calculated in $\mathrm{R}_{\mathrm{t}}$.

\subsection{Cubic Spline Interpolation}

Aiming at the complexity of the research and analysis of sustainable influence factors, the author applies the advanced mathematical theory of piecewise linear interpolation to solve the existing problems [66].

Definition 4. $a=x_{0}<x_{1}<\cdots<x_{n-1}<x_{n}=b$ division of a given interval $[a, b]$, If the function $S(x)$ satisfies: (1) It is a third-degree polynomial in each interval; (2) Each inner node has a secondorder continuous derivative; (3) $S\left(x_{i}\right)=y_{i}$, Then, $S(x)$ is called cubic spline interpolation function in the interval, which is: $S_{i}(x)=a_{i 0}+a_{i 1} x+a_{i 2} x^{2}+a_{i 3} x^{3} i=0,1, \cdots, n-1$.

Hypothesis $S^{\prime \prime}\left(x_{i}\right)=M_{i}(i=0,1, \cdots, n)$ in subinterval $\left[x_{i-1}, x_{i}\right]$ is a linear function of $x$, you can obtain $S^{\prime \prime}\left(, x_{i-1}\right)=M_{i-1}, S^{\prime \prime}\left(x_{i}\right)=M_{i} x=\left[x_{i-1}, x_{i}\right]$.

$S^{\prime \prime}(x)=M_{i-1} \frac{x_{i}-x}{x_{i-1}-x_{i}}+M_{i} \frac{x-x_{i-1}}{x_{i}-x_{i-1}}$, Get two points in a row:

$$
\left\{\begin{array}{c}
S_{i}(x)=M_{i-1} \frac{\left(x_{i}-x\right)^{3}}{6 h_{i}}+M_{i} \frac{\left(x-x_{i-1}\right)^{3}}{6 h_{i}}+A_{i}\left(x_{i}-x\right)+B_{i}\left(x-x_{i-1}\right) \\
S\left(x_{i-1}\right)=\frac{1}{6} M_{i-1} h_{i}^{2}+A_{i} h_{i}=f\left(x_{i-1}\right) \\
S\left(x_{i}\right)=\frac{1}{6} M_{i} h_{i}^{2}+A_{i} h_{i}=f\left(x_{i}\right)
\end{array}\right.
$$

Then, one obtains:

$$
S_{i}(x)=M_{i-1} \frac{\left(x_{i}-x\right)^{3}}{6 h_{i}}+M_{i} \frac{\left(x-x_{i-1}\right)^{3}}{6 h_{i}}+\left(y_{i-1}-\frac{M_{i-1}}{6} h_{i}{ }^{2}\right) \frac{\left(x_{i}-x\right)}{h_{i}}+\left(y_{i}-\frac{M_{i}}{6} h_{i}{ }^{2}\right) \frac{\left(x-x_{i-1}\right)}{h_{i}}\left(x \in\left[x_{i-1}, x_{i}\right], i=1,2, \cdots n\right)
$$


The above determines the $n+1$ values of $M_{0}, M_{1} \cdots M_{n}$, Determine the interpolation function $s(x)$, and obtain the derivative of commonly used boundary conditions:

Determining the linear equations of $M_{0}, M_{1} \cdots M_{n}$, the first boundary condition:

$$
\left[\begin{array}{ccccc}
2 & 1 & 0 & 0 & 0 \\
\mu_{1} & 2 & \lambda_{1} & 0 & 0 \\
0 & \ddots & \ddots & \ddots & 0 \\
0 & 0 & \mu_{n-1} & 2 & \lambda_{n-1} \\
0 & 0 & 0 & 1 & 2
\end{array}\right]\left[\begin{array}{c}
M_{0} \\
M_{1} \\
\vdots \\
M_{n-1} \\
M_{n}
\end{array}\right]=\left[\begin{array}{c}
g_{0} \\
g_{1} \\
\vdots \\
g_{n-1} \\
g_{n}
\end{array}\right]
$$

The second boundary condition:

$$
\left[\begin{array}{ccccc}
2 & \lambda_{1} & 0 & 0 & 0 \\
\mu_{2} & 2 & \lambda_{2} & 0 & 0 \\
0 & \ddots & \ddots & \ddots & 0 \\
0 & 0 & \mu_{n-2} & 2 & \lambda_{n-2} \\
0 & 0 & 0 & \mu_{n-1} & 2
\end{array}\right]\left[\begin{array}{c}
M_{1} \\
M_{2} \\
\vdots \\
M_{n-2} \\
M_{n-1}
\end{array}\right]=\left[\begin{array}{c}
g_{1}-\mu_{1} y_{0}{ }^{\prime \prime} \\
g_{2} \\
\vdots \\
g_{n-2} \\
g_{n-1}-\lambda_{n-1} y_{n}{ }^{\prime \prime}
\end{array}\right]
$$

The third boundary condition:

$$
\left[\begin{array}{ccccc}
2 & \lambda_{1} & 0 & 0 & 0 \\
\mu_{2} & 2 & \lambda_{2} & 0 & 0 \\
\vdots & \ddots & \ddots & \ddots & 0 \\
0 & 0 & \mu_{n-1} & 2 & \lambda_{n-1} \\
\lambda_{n} & 0 & 0 & \mu_{n} & 2
\end{array}\right]\left[\begin{array}{c}
M_{1} \\
M_{2} \\
\vdots \\
M_{n-1} \\
M_{n}
\end{array}\right]=\left[\begin{array}{c}
g_{1} \\
g_{2} \\
\vdots \\
g_{n-1} \\
g_{n}
\end{array}\right]
$$

The coefficient matrix of the equation system is non-singular and can be contacted with $\mathrm{M}_{0}, \mathrm{M}_{1} \cdots \mathrm{M}_{\mathrm{n}}$. Finally, the value and range of the variable $\mathrm{x}$ are obtained.

\section{Case Analysis and Management Design}

A successful project signifies that there is an optimal project management model used for dealing with problems and implementing various processes, and which ultimately achieves high economic benefits [67].

The good performance of project management depends on the optimal balance between safety, schedule, cost, quality, resources, and environmental impact [68-70]. A multiobjective design model is built for many impact factors. The weighted sum is calculated based on the designed project management model, and the priority ranking is determined to finally select a reasonable project management model.

\subsection{Case Characteristics Analysis}

Ling Jiang Bridge, an extra-long river-crossing bridge in the bid section of the Tai Zhou-Jin Hua Expressway in Zhe Jiang, China, was selected as the case for analysis. The bridge consists of the main bridge and the auxiliary bridge, with a total length of $1421.07 \mathrm{~m}$. The total length of the auxiliary bridge is $781.07 \mathrm{~m}$ (From 0\# 17\#, 22\# 25\#). The main beam comprises a concrete T-shaped beam and built-up box girder with continuous prestress poured on the $50 \mathrm{~m}$ and $25 \mathrm{~m}$ simply supported span beams (Figures 6 and 7). The main bridge is a five-span low tower cable-stayed prestressed concrete bridge with four towers and a single cable plane, and the bridge length is $640 \mathrm{~m}$. The main tower and beam body are consolidated, and the pier columns are separated from the main beam, except for the two pier columns in the middle. The main beam adopts a box-shaped section. The beam height at the top of the main pier is $5.5 \mathrm{~m}$, and the beam height at the top of the side pier and the middle pier is $3.0 \mathrm{~m}$. The top of the box girder is $27.0 \mathrm{~m}$ wide; the cantilever of a single side is $5.0 \mathrm{~m}$ long; the bottom of the box girder at the midspan is $15.8 \mathrm{~m}$ wide; the bottom of the box girder at the root is $14.27 \mathrm{~m}$ wide. The web outside the box girder is an 
angle web with a thickness of $50 \mathrm{~cm}$; the web inside the box girder is a straight web with a thickness of $45 \mathrm{~cm}$; the top web is $28 \mathrm{~cm}$ thick (Figure 6).

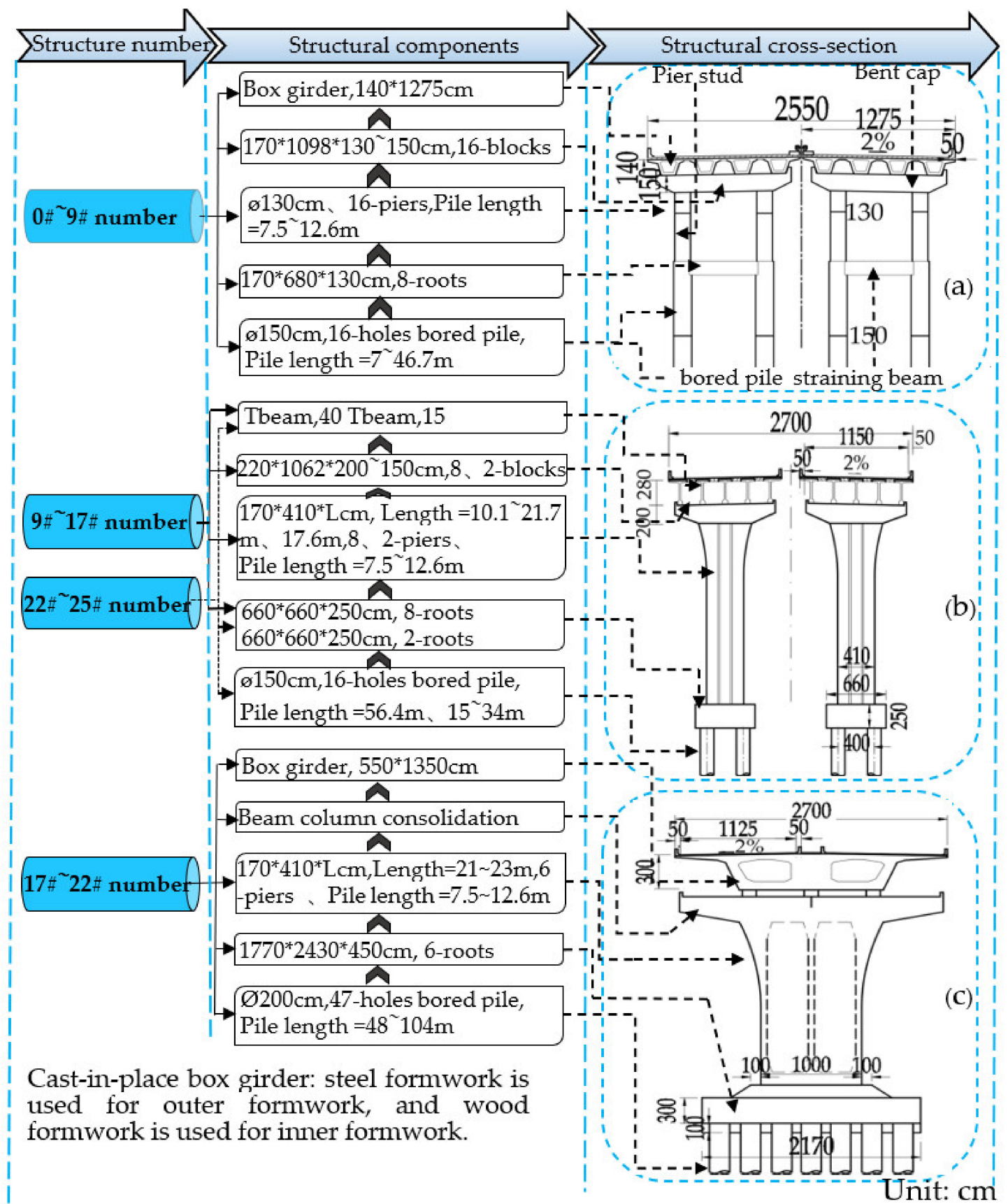

Figure 6. Structure distribution of cable-stayed bridge (0\# 25\#; cross-sectional view): (a) The 0\# 9\# structure is prestressed concrete small box girder; (b) The 9\# 17\# and 22\# 25\# structures are prestressed concrete T-beam; (c) The 17\# 22\# structure is large box beam of prestressed concrete. 


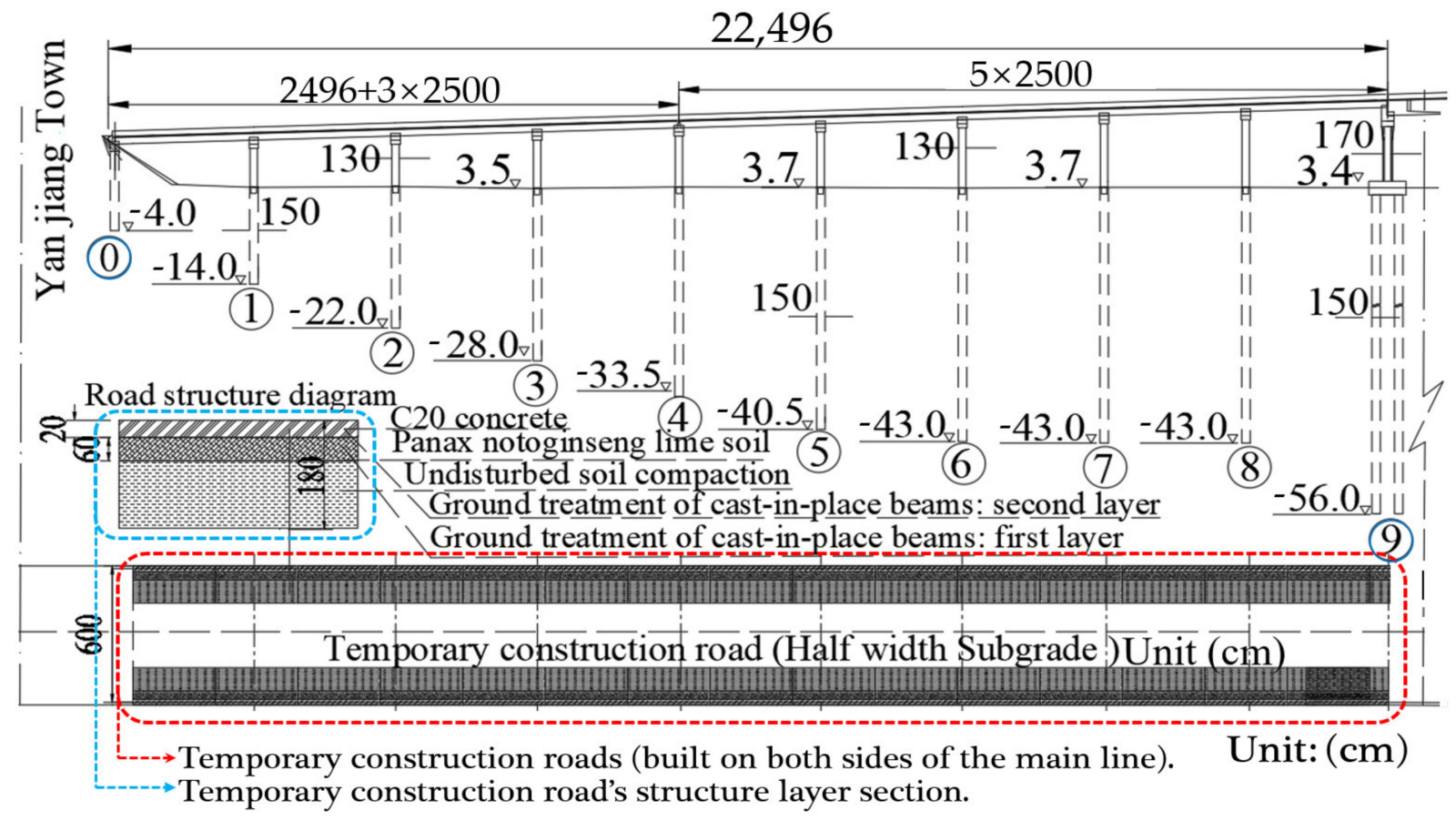

(a)

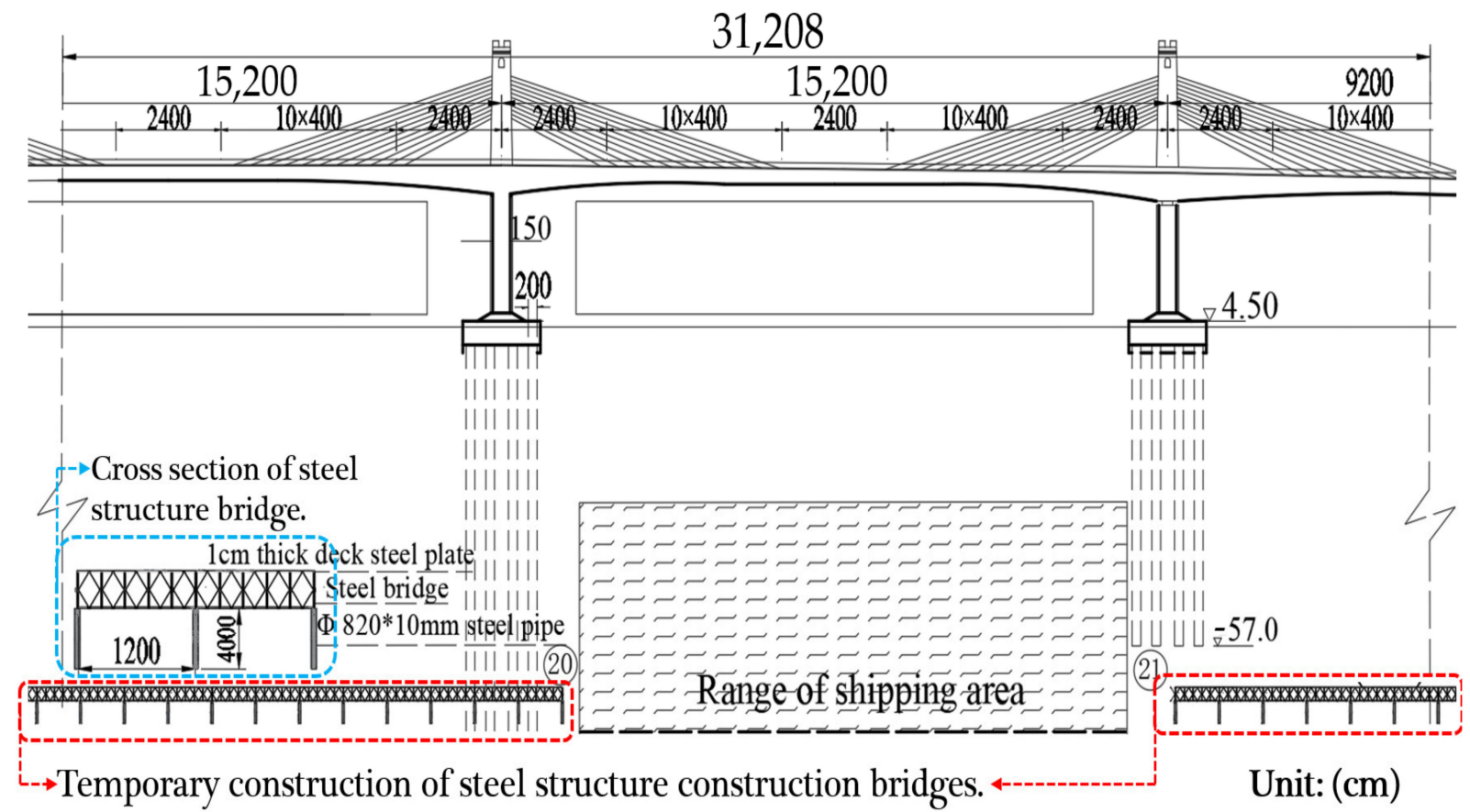

(b)

Figure 7. Organization and management plan of construction bridge project: (a) Temporary construction of temporary bridge (part); (b) Temporary construction of steel structure bridge (part).

The construction period of the cable-stayed bridge is 1247 days. The difficulties of project management are as follows:

- The construction site is narrow and spans the Ling Jiang River, which has an average tidal range of $4.02 \mathrm{~m}$. During the construction period, it is necessary to ensure the normal navigation of 1000-tonne maritime ships. The local Bureau of Maritime Affairs approved the construction period of the main bridge to be 913 days. 
- The construction site is affected by the natural climate in much of the year. The Mei Yu flood season is from March to June, and the typhoon season is from July to September.

- The bridge structure is complex; the construction of supporting auxiliary facilities uses up the construction period.

- Many workers engaging in professional types of work are required because of the short construction period, resulting in high risks for personnel management.

- Experienced management and technical personnel are required, such as full-time safety, power, structure, construction, quality, and test engineers, due to the difficulties relating to safety, progress, and quality management.

The project management tasks were organized into and implemented as four sections based on the progress (Figure 6): 0\# 9\#, 9\# 17\#, 17\# 22\#, 22\# 25\#. The project manager responsibility system was adopted for the project. Outstanding teams and leaders should be equipped with a wealth of organizational skills, coping skills, team skills, leadership and team building skills, communication skills and technical skills [71].

\subsection{Project Management Planning and Design}

In 2012, China accelerated the implementation model of green building development. In 2013, a green house action plan was released to promote government investment in green buildings and the sustainable development of green housing [72]. In the optimal design of bridge project management, the environmental impact should be taken as the top priority, followed by the project construction cost. The core parameters of the environmental impact are selected based on the five factors determined by the ISO: particulate matter formation potential, fume, and dust; acidification potential; free-water eutrophication potential; global warming potential; and waste potential [56].

As shown in Figure 8, the project management organization system for the cablestayed bridge and the composition of the supporting facilities poses great challenges for the environmental and economic cost. A total of 709 management staff and skilled workers participated in the project. The peak period of construction lasted from October 2007 to August 2010, about 1060 days (including the typhoon period).

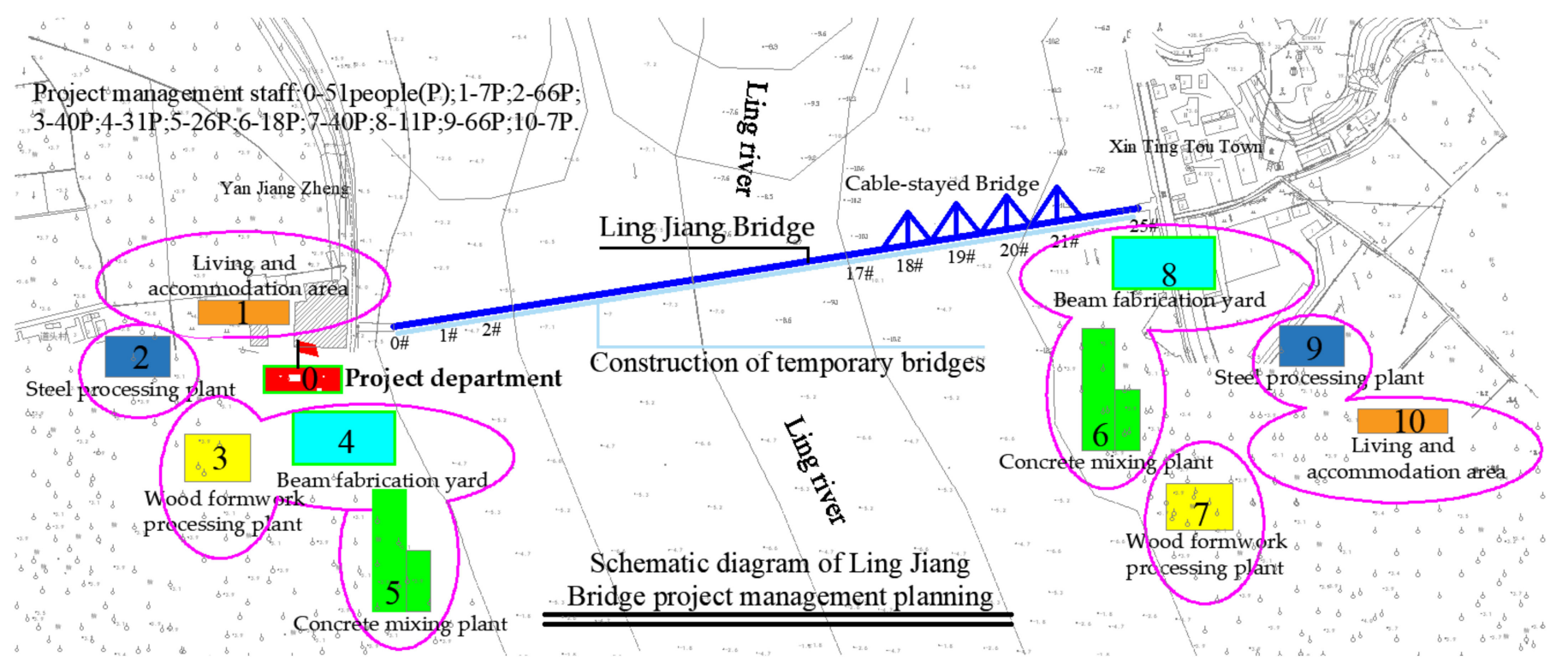

Figure 8. The project management organization's arrangement of on-site planning.

Four project management models were designed (Figure 9) according to the design drawings, construction organization design, Technical Specification for Construction of Highway Bridges and Culverts (JTJ 041-2000), and Code for Pile Foundation of Harbor Engineering (JTJ 254-98) [73]. The construction of auxiliary facilities, the organization 
of construction personnel and construction groups, and the arrangement of mechanical equipment were all subject to substantial changes with the adjustment of the management model. To ensure the normal navigation of ships on the Ling Jiang River, the main beam between bridge sites 20\# and 21\# was installed by the hanging basket method, and other management models were not adopted. Four sets of hanging baskets were installed at bridge sites 18\#,19\#, 20\# and 21\# for symmetric construction. Each span of the main bridge is $152 \mathrm{~m}$ long, which was divided into 20 symmetric blocks (including 0\# 19\# and the closure section) for construction. The construction period of each block was not to be longer than 18 days; otherwise, the total construction period and schedule of the entire bridge would be affected.

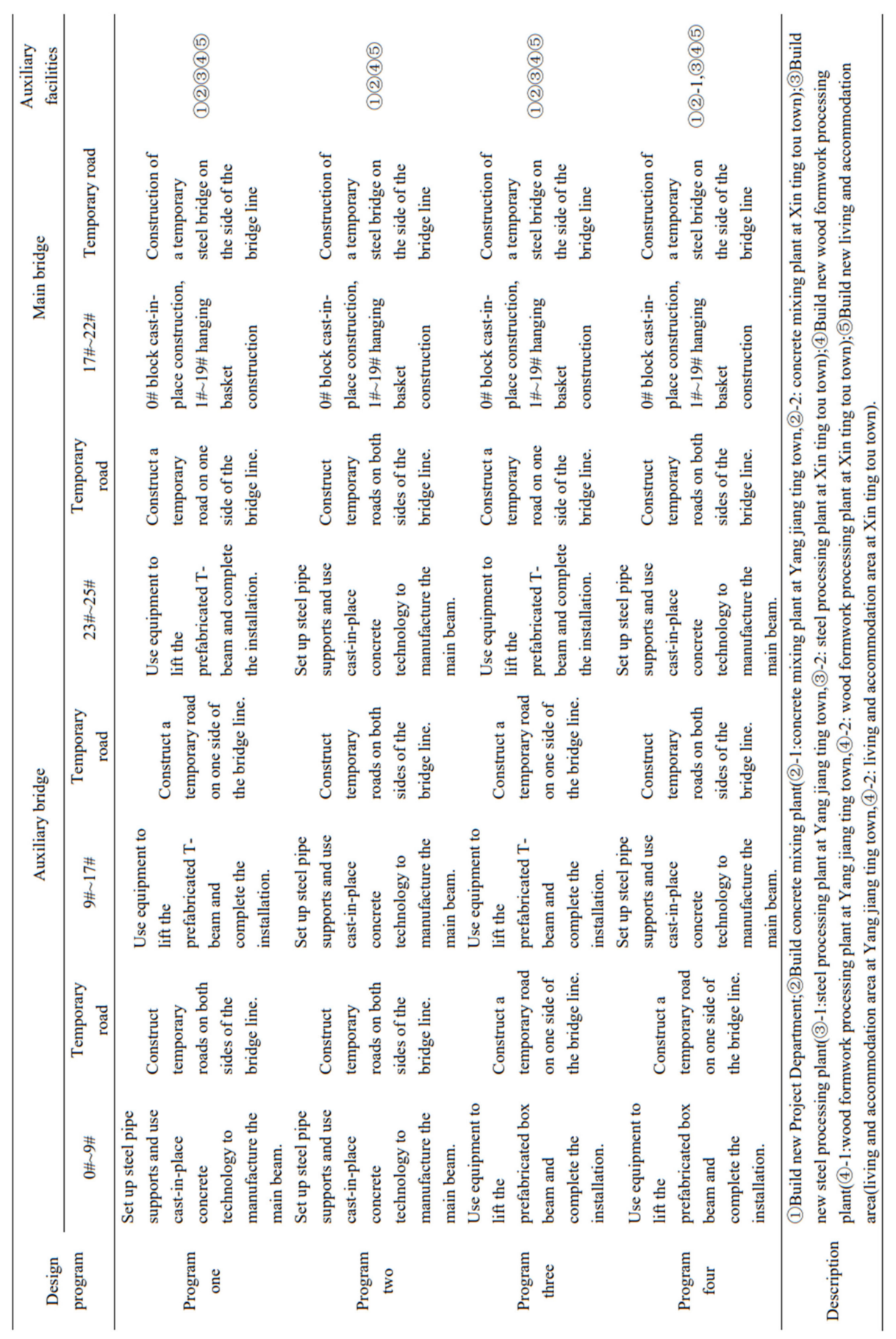

Figure 9. Description of the overall project management plan. 
Each bridge site was arranged with one tower crane with an arm span of $75 \mathrm{~m}$ for the hoisting work. The auxiliary bridge is located at both sides of Ling Jiang River. The shallow bedrock and the stable foundation were able to meet the conditions for erecting the cast-in situ main beams by full scaffold. T-shaped beams and box girders were erected by two 200 -tonne truck cranes. The main beams were transported by the beam-carrying vehicle to the site of erection. The outside of the bridge area was built with temporary roads to meet the traffic requirements (Figures 7 and 9).

\section{Results and Discussion}

Table 3 shows that the environmental pollution of the bridge during the project management mainly derives from the manufacturing of raw materials. The steel production generates $62,622.55$ tonnes of pollution, followed by $27,508.29$ tonnes generated by the production of cement. The construction cost of the main bridge is higher than that of the auxiliary bridge. The common feature of the two parts is that the costs of the reinforcement bars and C account for a high proportion of the total cost, at $46.09 \%$ and $65.71 \%$, respectively. Specifically, the costs of the reinforcement bars of the two parts are CNY 37.122 million and CNY 18.014 million; the costs of the C of the two parts are CNY 5.056 million and CNY 2.618 million.

Table 3. Bridge environmental impact and economic cost.

\begin{tabular}{cccccc}
\hline Project Name & Number (Tonne) & Ratio (\%) & Project Name & Cost Incurred (CNY) & Ratio (\%) \\
\hline Material preparation stage & $164,038.23$ & 57.11 & Main bridge & $190,231,873$ & 68.56 \\
Construction stage & $86,384.14$ & 30.08 & Vice bridge & $67,245,171.51$ & 24.23 \\
(1) & $10,931.43$ & 3.81 & $(1)$ & $1,433,139.42$ & 0.52 \\
$(2)-1$ & 3310.94 & 1.15 & $25-1$ & $5,740,563.26$ & 2.07 \\
(2)-2 & 3310.94 & 1.15 & $(2)-2$ & $4,029,419.28$ & 1.45 \\
(3)-1 & 4030.53 & 1.4 & $(3)-1$ & $3,737,910.48$ & 0.95 \\
(3)-2 & 2777.80 & 0.97 & $(3)-2$ & $2,627,562.34$ & 0.31 \\
(4)-1 & 2033.43 & 0.71 & $(4)-1$ & 870,203 & 0.27 \\
(4)-2 & 1530.26 & 0.53 & $(4)-2$ & $739,672.55$ & 0.15 \\
(5)-1 & 6458.23 & 2.25 & $(5)-1$ & $410,046.44$ & 0.15 \\
(5)-2 & 2418.26 & 0.84 & $(5)-2$ & $410,046.44$ & \\
\hline
\end{tabular}

Figure 10, the 11 subprojects of the main bridge have the highest environmental impacts and economic costs, as well as which the GWP has the largest value in terms of the environmental impact, which is greater than the values of other influencing factors. At the same time, it can be found that the auxiliary subprojects in the project management have a very important impact on the sustainable development goals. The environmental impact accounts for $1.72 \%$ of the total, and the economic cost accounts for $7.21 \%$ of the total price.

The analysis of all stages of the bridge project management was completed, and the designed values of the four models were evaluated according to Figure 9 as the basis for judging the optimum project management model. 


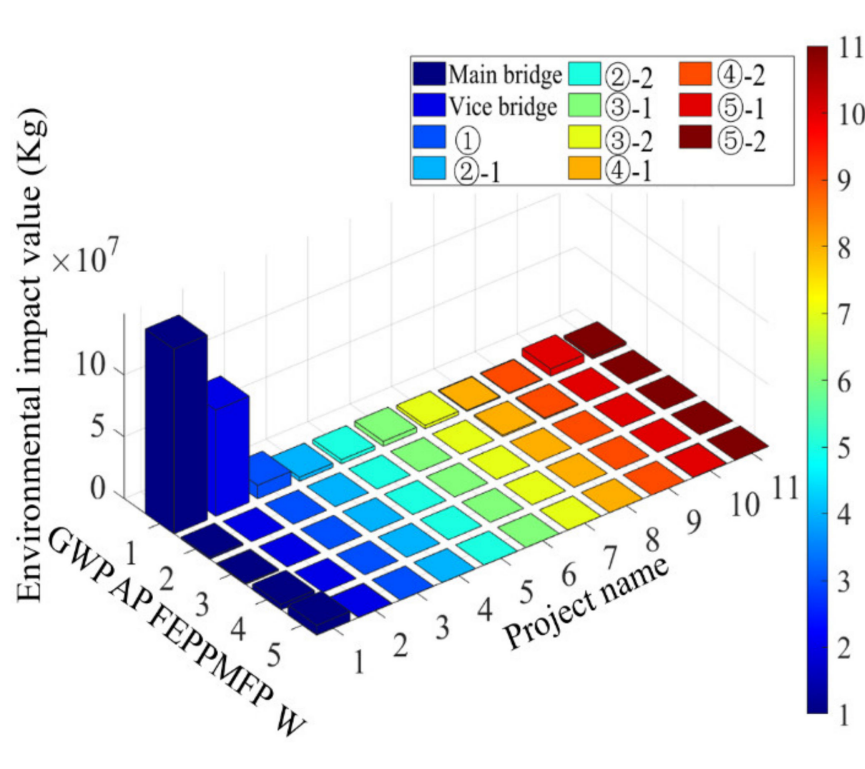

(a)

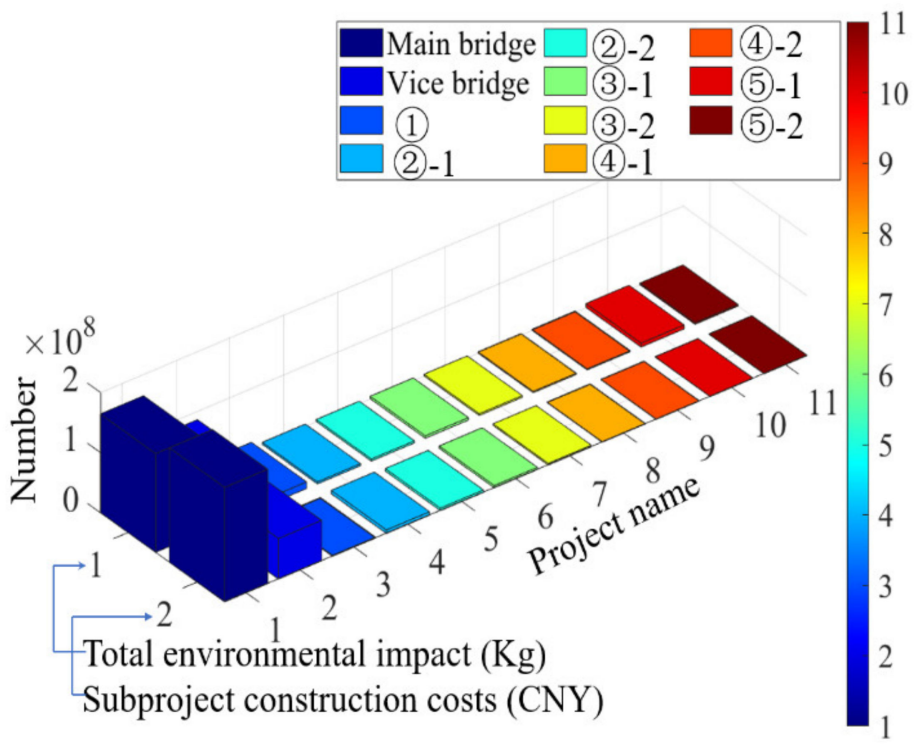

(b)

Figure 10. Subproject analysis: (a) The environmental impact of the subprojects; (b) The economic cost values of the subprojects.

According to the design model, the construction of temporary roads and the treatment of the ground foundation of the cast-in-place beam bodies need to be completed before the foundation construction and the main beam construction are started; the temporary roads were designed in two parts:

- For sections $0 \# \sim 17 \#$ and 22\# 25\#, the original ground was compacted within a depth of $100 \mathrm{~cm}$; the compacted surface is compacted layer by layer and then backfilled with $60 \mathrm{~cm}$ of 37 lime earth, and a $20 \mathrm{~cm}$ layer of C20 was poured on the lime earth.

- For sections 17\# 20\# and 21\# 23\#, a temporary steel bridge was built as a temporary passage. The foundation of the temporary steel bridge was made of $\varnothing 820 \mathrm{~mm} \times 10 \mathrm{~mm}$ steel pipes with a length of $3500 \sim 4000 \mathrm{~cm}$. The pile driver was pressed into the stressed rock stratum to a depth of $50 \sim 80 \mathrm{~cm}$ in the river. The steel pipe piles were arranged in two rows, with a vertical spacing of $1200 \mathrm{~cm}$ and a horizontal spacing of $400 \mathrm{~cm}$ (Figure 6).

As shown in Figure 11 (See Section 3.4 for scientific algorithm theory), the curve analysis of the environmental impact value and economic cost value analyzed by Models 1 and 2 (using Matlab scientific algorithm programming program [74], the quadratic equation is obtained as $Z=(4.168) \times x+\left(-1.117 \times 10^{-5}\right) \times x^{2}+\left(-1.094 \times 10^{4}\right)$. Drawing the surface analysis diagram, it can be concluded that the linearity of the sustainable development data of models 1 and 2 is basically the same, first increasing and then decreasing, showing the change of the quadratic parabolic equation. According to the obtained surface equations, biharmonic spline curve interpolation approximation analysis is carried out. $\mathrm{f}(\mathrm{x}, \mathrm{y})=$ double harmonic surface calculation $\mathrm{P}$; $\mathrm{x}$ is the mean value of $1.5 \times 10^{5}$ and std $8.803 \times 10^{4}$ normalized, $y$ is the mean value of $1.52 \times 10^{5}$ and std $8.803 \times 10^{4}$ normalized; coefficients: $p=$ coefficient structure; goodness of fit: SSE: $8.902 \times 10^{-19}$. The research conclusion is consistent with the curved surface conclusion. 


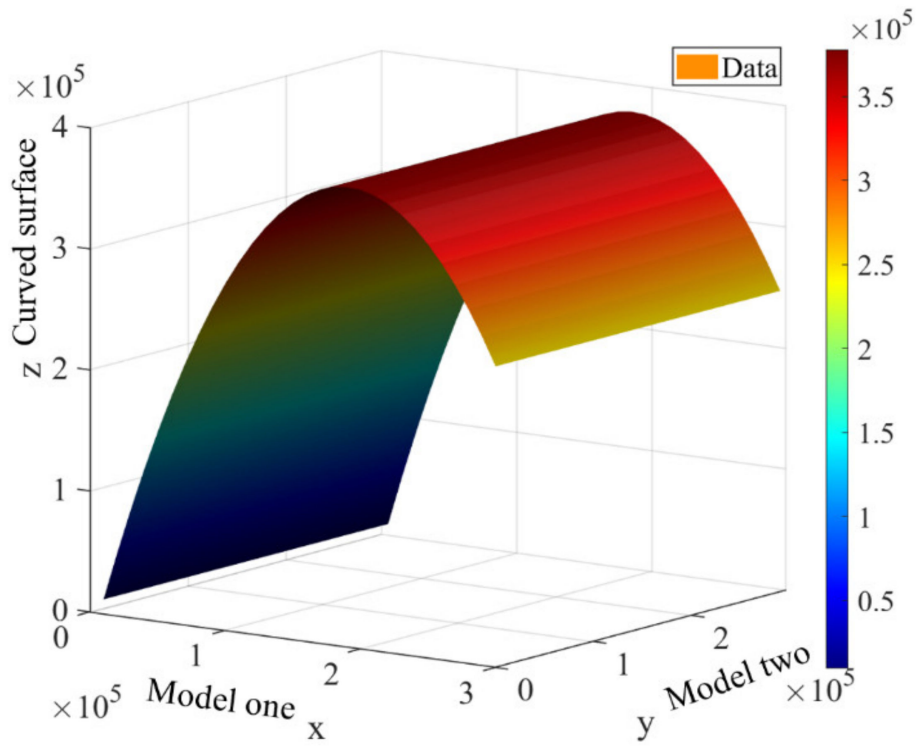

(a)

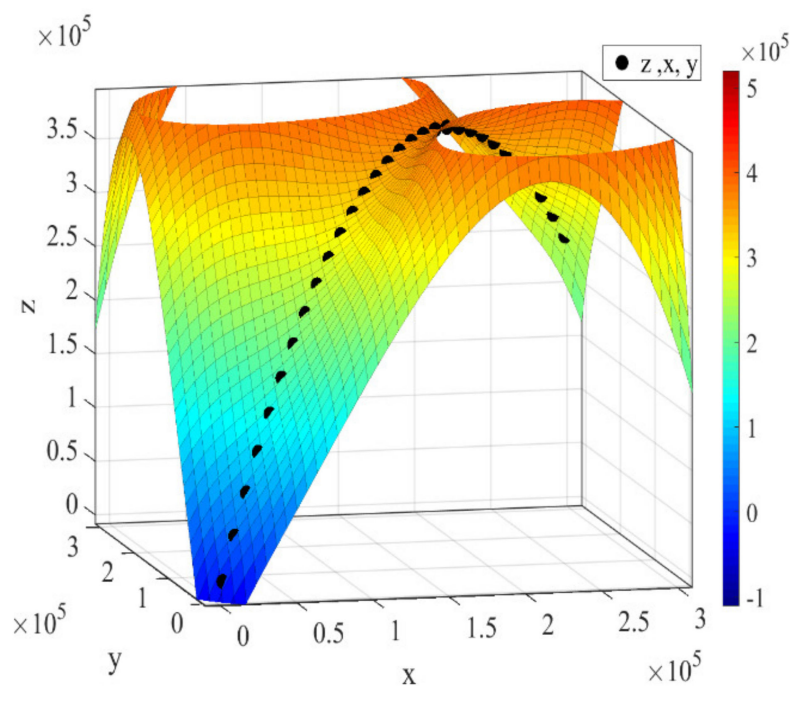

(b)

Figure 11. Model analyses 1 and 2: (a) Environmental impact and economic cost curved surface analysis; (b) Interpolation approximation analysis.

Figure 12 shows the surface analysis of models 3 and 4, and the quadratic equation is $\mathrm{z}=(-0.9432) \times \mathrm{x}+\left(5.769 \times 10^{-6}\right) \times \mathrm{x}^{2}+\left(8.472 \times 10^{4}\right)$. The initial value of surface judgment models 3 and 4 is greater than models 1 and 2 , and the final value of surface is smaller than models 1 and 2; biharmonic spline interpolant: $f(x, y)=$ biharmonic surface computed, $\mathrm{P}$ is calculated by double-harmonic surface, the average value of $\mathrm{x}$ is $1.48 \times$ $10^{5}$ and std $8.803 \times 10^{4}$ normalized, and the average of $\mathrm{y}$ is $1.51 \times 10^{5}$ and std $8.803 \times$ $10^{4}$ normalized; coefficient: $\mathrm{p}=$ coefficient structure; goodness of fit: SSE: $4.802 \times 10^{-20}$; R-squared: 1.

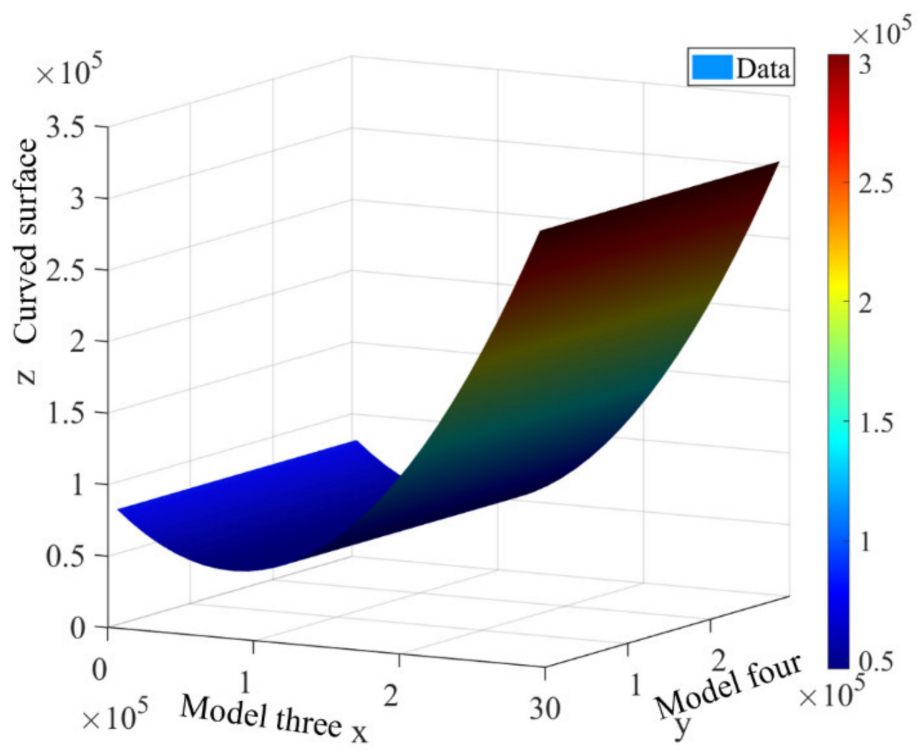

(a)

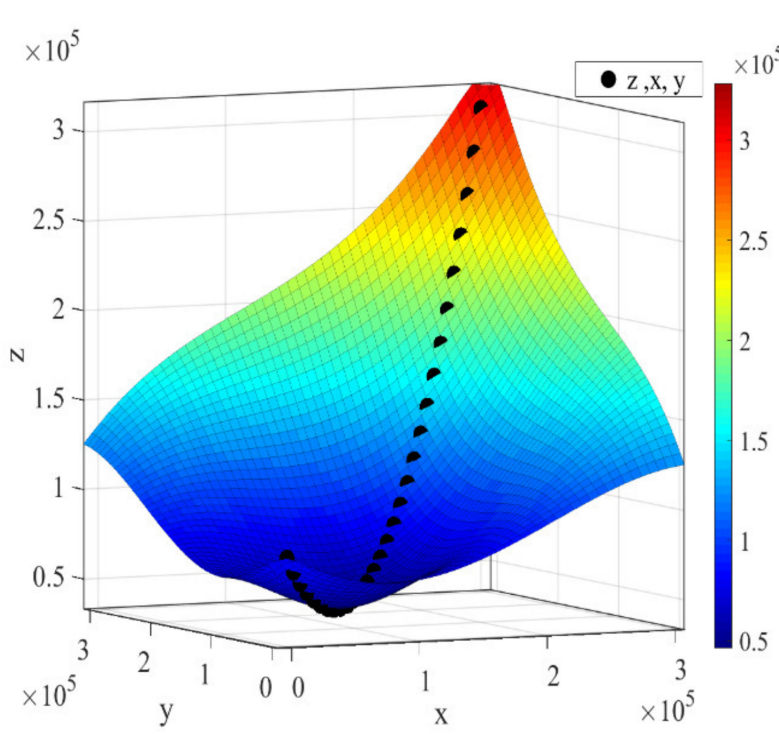

(b)

Figure 12. Model analyses 3 and 4: (a) Environmental impact and economic cost curved surface analysis; (b) Interpolation approximation analysis. 
The numerical value of model 3 is smaller than that of model 4 , and the analysis conclusion drawn is very consistent with the software numerical judgment conclusion.

\subsection{Algorithm Optimization Assessment}

Table 4 shows the environmental impact and construction costs caused by the foundation treatment of the supports for the construction of the main beams and the construction of roads on site. The environmental impacts of the four models are ranked as follows:

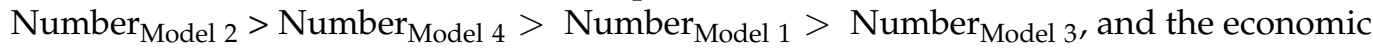
cost incurred is Cost $_{\text {Model } 2}>$ Cost $_{\text {Model 4 }}>$ Cost $_{\text {Model 1 }}>$ Cost $_{\text {Model 3 }}$. Based on these two indicators, Model 3 is determined as the optimum project management mode. The steel pipe support and temporary roadway data are shown in Table 5.

Table 4. Environmental contribution and economic cost of four design options.

\begin{tabular}{cccccc}
\hline \multirow{2}{*}{$\begin{array}{c}\text { Project Management } \\
\text { Model }\end{array}$} & \multicolumn{2}{c}{ Design Number } & \multicolumn{2}{c}{ Material and Construction Phase } & \multicolumn{2}{c}{ Increase Auxiliary Facilities } \\
\cline { 3 - 7 } & & EIN (Tonne) & CI (TC) & EIN (Tonne) & CI (TC) \\
\hline Model one & (1) (2) (3) (4)(5) & $287,224.18$ & $27,747.56$ & $65,830.38$ & 511.58 \\
Model two & (1) (3) (4) (5) & $280,602.31$ & $26,770.56$ & $218,480.51$ & 796.97 \\
Model three & (1) (2) (3) (4) (5) & $287,224.18$ & $27,747.56$ & 3092.05 & 388.74 \\
Model four & (1) (2)-1(3) (4) (5) & $283,913.25$ & $27,344.62$ & $155,742.18$ & 683.55 \\
\hline
\end{tabular}

Note: EIN = Environmental impact number; $\mathrm{CI}=$ Cost incurred; TC = Thousand CNY (Abbreviations are used in Table 4).

Table 5. Data of steel pipe support and temporary road.

\begin{tabular}{|c|c|c|c|c|c|c|c|c|c|}
\hline \multirow[b]{2}{*}{$\mathbf{P M}$} & \multirow[b]{2}{*}{ AF } & \multicolumn{2}{|c|}{ 0\# 9\# } & \multicolumn{2}{|c|}{ 9\# 17\# } & \multicolumn{2}{|c|}{ 17\# 22\# } & \multicolumn{2}{|c|}{ 22\# 25\# } \\
\hline & & $\begin{array}{c}\text { EIN } \\
\text { (Tonne) }\end{array}$ & CI (TC) & $\begin{array}{c}\text { EIN } \\
\text { (Tonne) }\end{array}$ & CI (TC) & $\begin{array}{c}\text { EIN } \\
\text { (Tonne) }\end{array}$ & CI (TC) & $\begin{array}{c}\text { EIN } \\
\text { (Tonne) }\end{array}$ & CI (TC) \\
\hline \multirow{2}{*}{$\begin{array}{l}\text { Model } \\
\text { one }\end{array}$} & SPB & $62,292.59$ & 93.37 & 1 & 1 & 1 & 1 & 1 & 1 \\
\hline & TR & 891.49 & 58.95 & 783.70 & 51.82 & 1561.74 & 2.87 .55 & 300.86 & 19.89 \\
\hline \multirow{2}{*}{$\begin{array}{l}\text { Model } \\
\text { two }\end{array}$} & SPB & $171,813.20$ & 2.40 .97 & 0 & 0 & 1 & 1 & $42,044.96$ & 66.08 \\
\hline & TR & 2458.89 & 1.62 .59 & 0 & 0 & 1561.74 & 2.87 .55 & 601.72 & 39.79 \\
\hline \multirow{2}{*}{$\begin{array}{l}\text { Model } \\
\text { three }\end{array}$} & SPB & 1 & 1 & 1 & 1 & 1 & 1 & 1 & 1 \\
\hline & TR & 1229.44 & 81.29 & & & 1561.74 & 2.87 .55 & 300.86 & 19.89 \\
\hline \multirow{2}{*}{$\begin{array}{l}\text { Model } \\
\text { four }\end{array}$} & SPB & 1 & 1 & $109,520.61$ & 1.57 .02 & 1 & 1 & $42,044.96$ & 66.08 \\
\hline & TR & 445.75 & 29.47 & 1567.39 & 1.03 .65 & 156.1.74 & 2.87 .55 & 601.72 & 39.79 \\
\hline
\end{tabular}

Note: $\mathrm{PM}=$ Project management model; $\mathrm{AF}=$ Auxiliary facilities; $\mathrm{SPB}=$ Steel pipe bracket; TR = Temporary Road; EIN = Environmental impact number; $\mathrm{CI}=$ Cost incurred; $\mathrm{TC}=$ Thousand CNY (Abbreviations are used in Table 5).

According to Formulas (3), (11) and Table 3, the membership matrix of project evaluation in construction stage is established:

$$
P(u)=\left[\begin{array}{cc}
p_{\mu_{p 1}} & 0 \leq \mu_{\mathrm{p} 1} \leq 1 \\
p_{\mu_{p 2}} & 0 \leq \mu_{\mathrm{p} 2} \leq 1 \\
\vdots & \vdots \\
\vdots & \vdots \\
p_{\mu_{p n}} & 0 \leq \mu_{\mathrm{pn}} \leq 1
\end{array}\right]=\left[\begin{array}{cccccc}
0.5783 & 0 & 0 & 0 & 0 & 0 \\
0 & 0.3045 & 0 & 0 & 0 & 0 \\
0 & 0 & 0.024 & 0 & 0 & 0 \\
0 & 0 & 0 & 0.0233 & 0 & 0 \\
0 & 0 & 0 & 0 & 0.0313 & 0 \\
0 & 0 & 0 & 0 & 0 & 0.0385
\end{array}\right] \text {, }
$$

(The degree of membership of the six influencing factors.)

Figure 13 shows the project management organization of the bridge has a discrete relationship between environmental impact and economic cost. The designed project management model can reduce the environmental pollution by $208,766.58$ tonnes, accounting for $71.91 \%$ of the total amount after the design, and reduce the construction cost by CNY 6.916 million, accounting for $2.51 \%$ of the total design cost. 


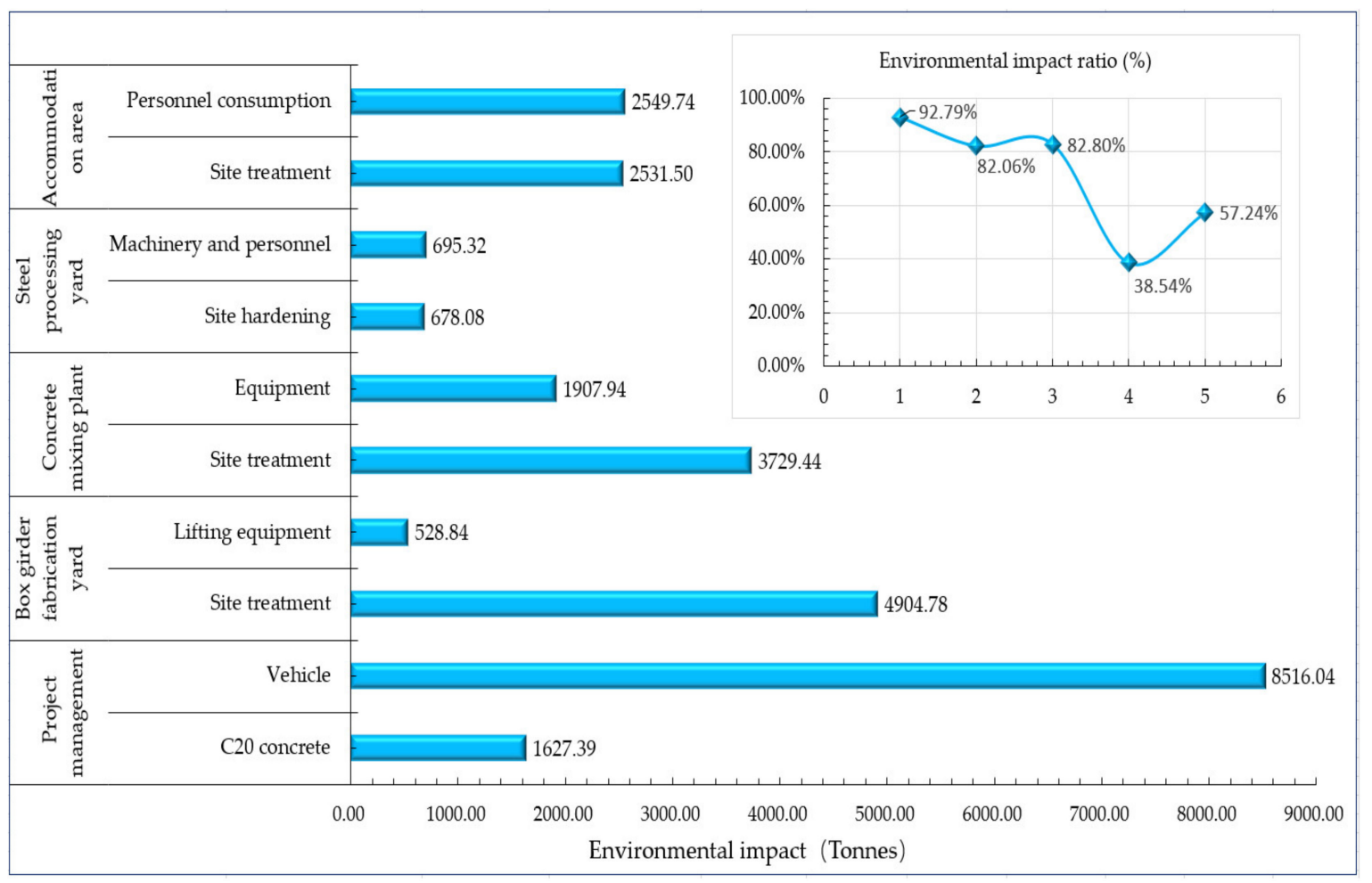

(a)

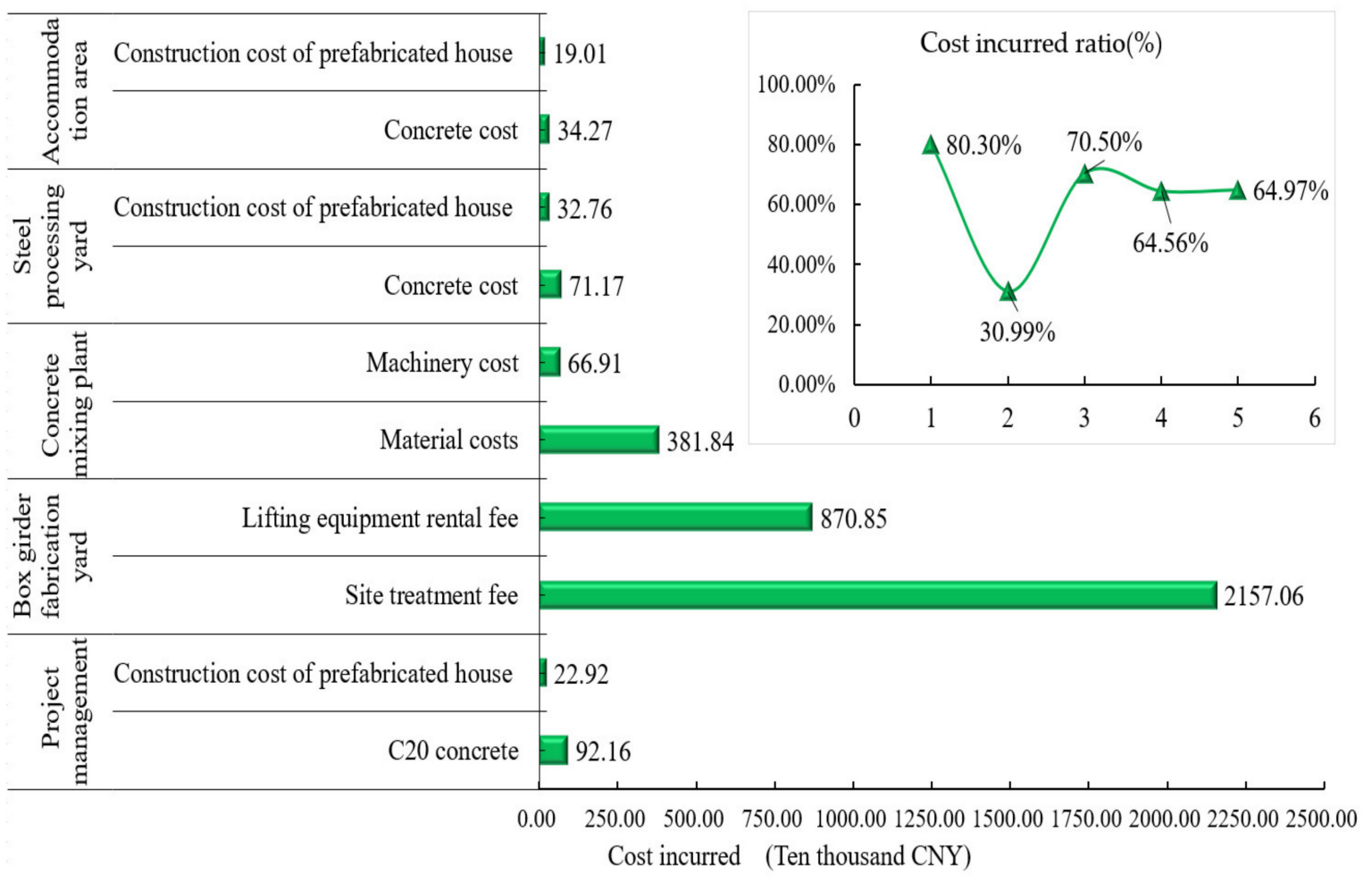

(b)

Figure 13. Analysis of auxiliary facilities: (a) Environmental impact; (b) The economic cost.

Figure 14 shows the piecewise cubic interpolation performed for three types of influencing factors: $f(x, y)=$ use cubic segmented surface to calculate $P$; $x$ is normalized with 
mean 3 and std 1.581; y is normalized with mean 0.708 and std 0.2219; SSE: 0 (The sum variance is 0 , indicating that the data fitting is very accurate). The fitting conclusion is that the steel processing yard and accommodation area have the lowest index value among environmental impact and economic impact. The five stages are discretely distributed.

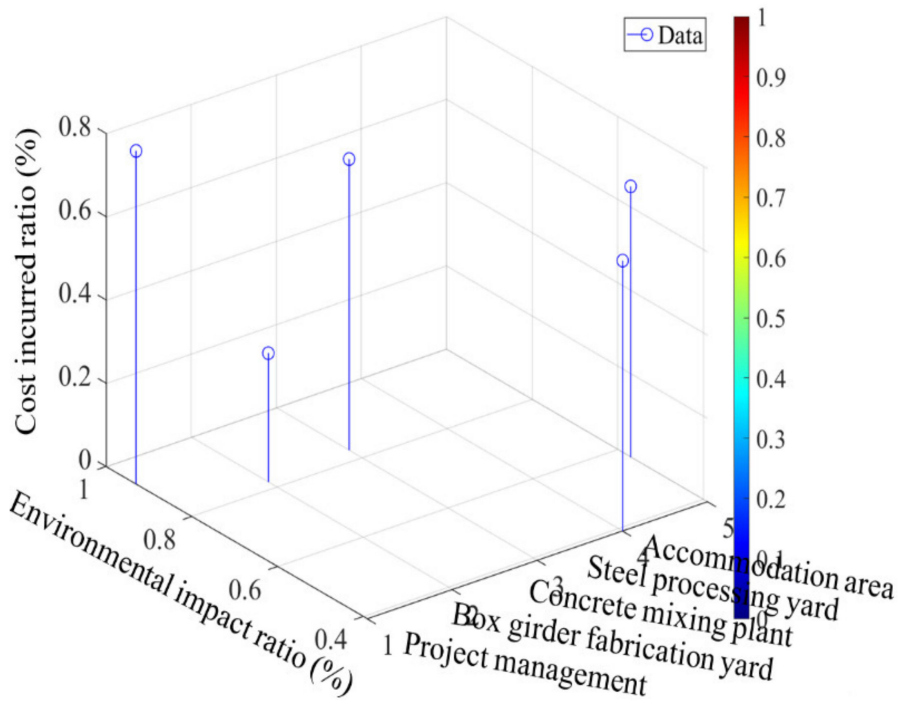

(a)

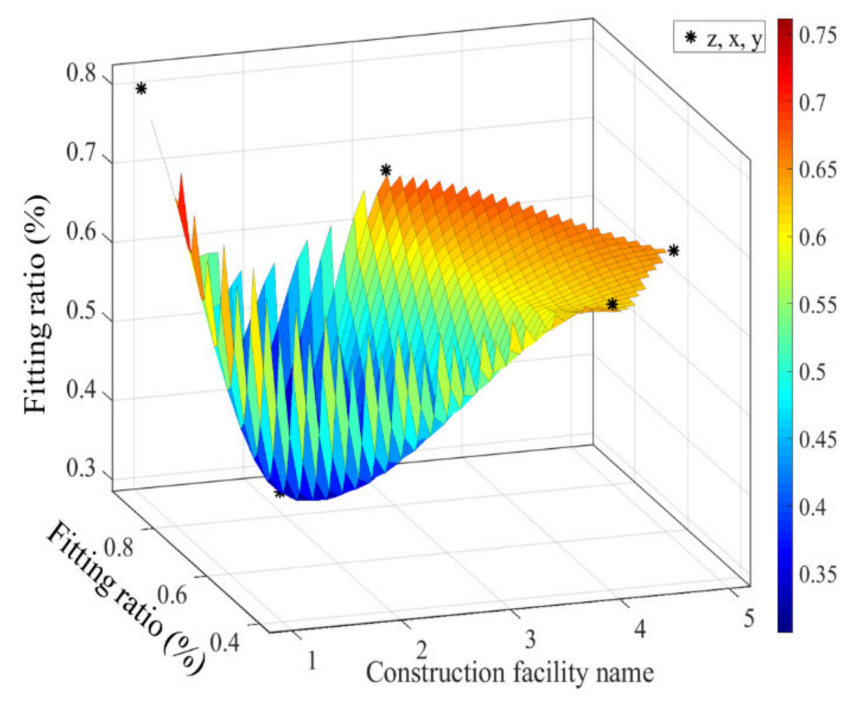

(b)

Figure 14. Ancillary facilities: (a) The environmental impact and economic cost; (b) Interpolation analysis of environmental impact and economic cost.

Figure 15 shows the environmental impact and economic cost of the different project management modes. The comparison chart was drawn using the impact factor product method. Assuming Model 3 = K, we can obtain Model $1=1.22 \mathrm{~K}$, Model $4=1.50 \mathrm{~K}$, and Model $2=1.69 \mathrm{~K}$, and the order is Model 3 $\rightarrow$ Model $1 \rightarrow$ Model $4 \rightarrow$ Model 2.

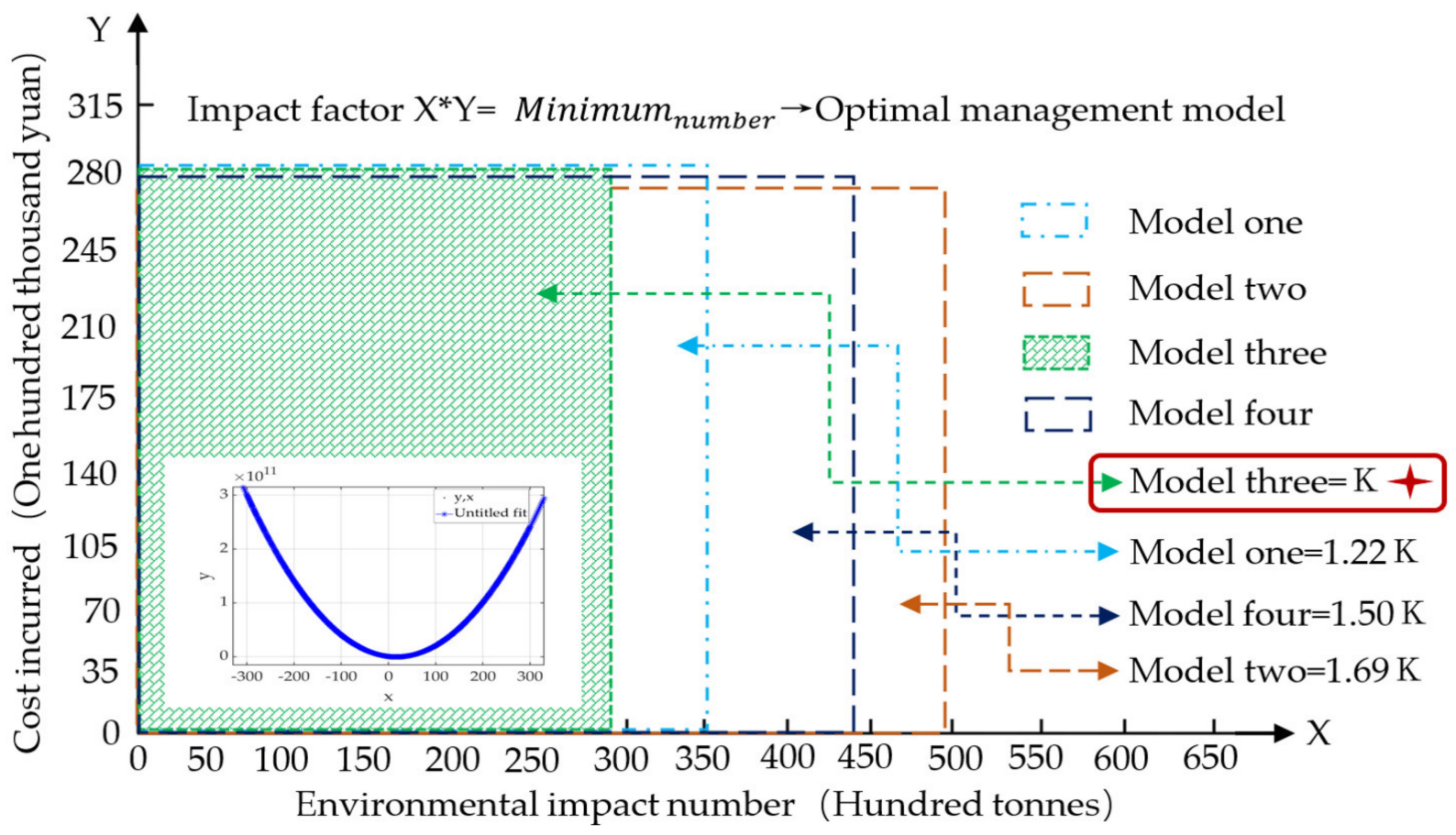

Figure 15. Comparison of the environmental impact and economic cost of the four options after comprehensive assessment (Figure x-y: Model 3 Interpolation analysis, the conclusion is that the mean is symmetric). 
The results show that Model 3 is the best in terms of environmental impact and economic cost, and it is also the best management mode. As shown in the parabolic diagram of Model 3, the values of $\mathrm{x}$ and $\mathrm{y}$ tend to be symmetrical on the $\mathrm{y}$ axis and distributed uniformly.

Figure 16 shows that the conclusion of the logarithmic function equation analysis is consistent with the data calculation; Model 3 is the best project management model.

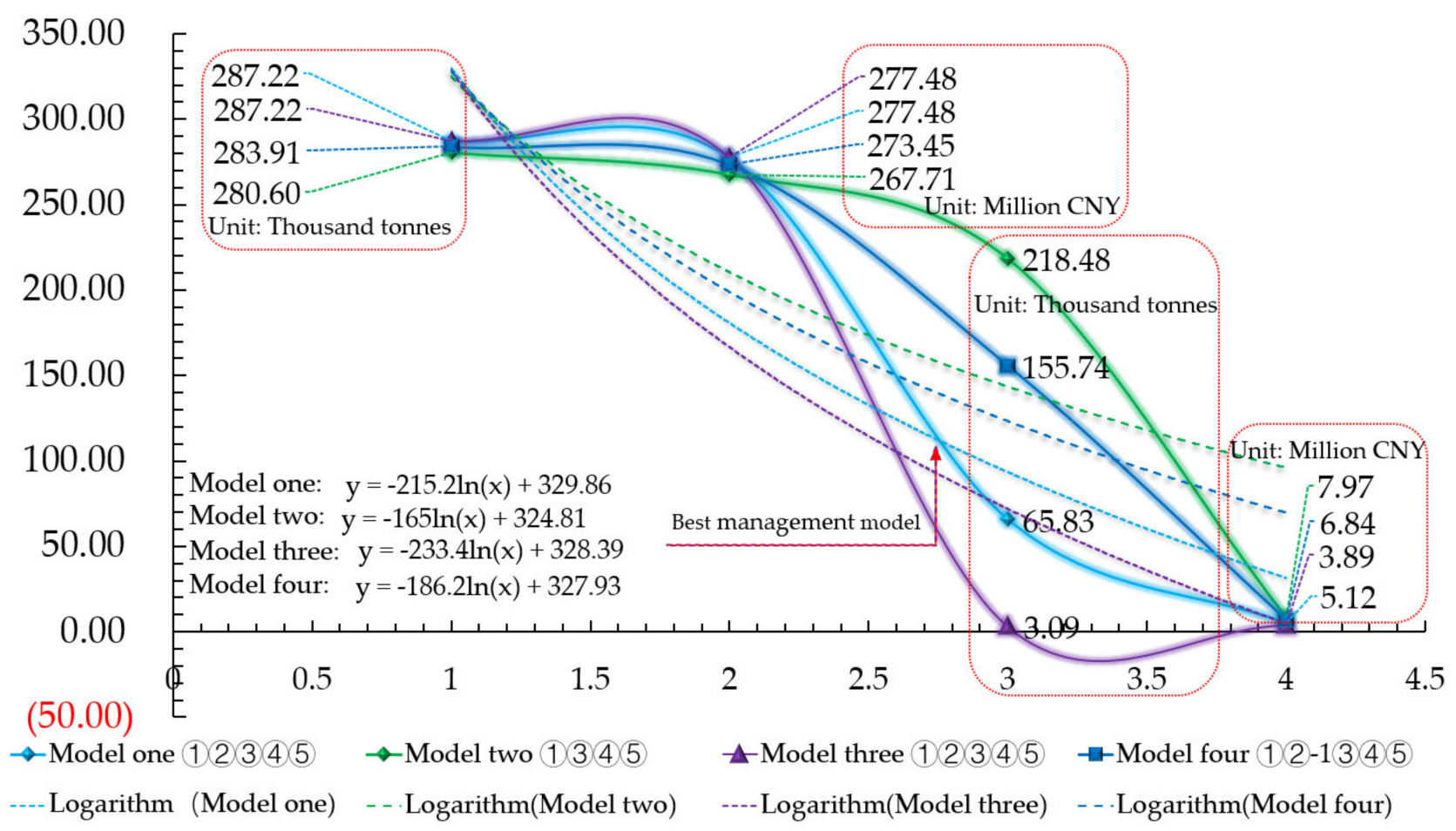

Figure 16. Comparative analysis of four model curve data.

As shown in Figure 17 (see Section 3.4 for the algorithm), according to the quadratic equations of the four models obtained in Figure 16, the nearest neighbor interpolation fitting is performed and the optimal model is determined by the Matlab scientific algorithm [75].

Quadratic equations of the four models:

- Model 1: $f(x, y)=(-4 e+07) \cdot{ }^{*} x+(-1 e+07) .{ }^{*} x \cdot{ }^{\wedge} 2+(4 e+08)$.

- Model 2: $f(x, z)=(2 e+08) \cdot{ }^{*} x+(-5 e+07) \cdot{ }^{*} x \cdot{ }^{*} 2+(2 e+08)$.

- Model 3: $f(x, k)=(-1 e+08) .{ }^{*} x+(3 e+06) \cdot{ }^{*} x \cdot{ }^{*} 2+(4 e+08)$.

- Model 4: $f(x, n)=(8 e+07) \cdot{ }^{*} x+(-3 e+07) .{ }^{*} x \cdot{ }^{*} 2+(2 e+08)$.

$\mathrm{X}=$ Numerical interval for environmental impact and economic cost calculation; $\mathrm{y} ; \mathrm{z}$; $\mathrm{k} ; \mathrm{n}=$ Numerical interval of quadratic equation after fitting; $\mathrm{e}=10$ (scientific notation, for example: $-4 \mathrm{e}+07=-4 \times 10^{7}$ ).

The nearest neighbor interpolation criterion:

1. $\quad \mathrm{SSE}=0$ (the sum of squared errors of the corresponding points of the fitted data and the original data. The closer the SSE is to 0 , the more successful the data prediction).

2. The smaller the Std value (in the interval of $y, z, n, k$ ) the better the model.

Matlab calculation program:

$>>$ clear all; $\%$ The first set of analyses;

$>>x=[-50,000,000: 100,000: 350,000,000]$;

$>>y=(-4 e+07) \cdot{ }^{*} x+(-1 e+07) \cdot{ }^{*} x \cdot{ }^{\prime} 2+(4 e+08) ;$

$>>z=(2 e+08) \cdot{ }^{*} x+(-5 e+07) .{ }^{*} x \cdot{ }^{*} 2+(2 e+08) ;$

$>>\operatorname{stem} 3(\mathrm{x}, \mathrm{y}, \mathrm{z})$;

$>>\operatorname{cftool}(x, y, z)$;

Fitting conclusion: 
1a, In $f(x, k, n): x \in(1.5 e+08), \operatorname{Std} \in(1.155 e+08) ; n \in(1.075 e+23), \operatorname{Std} \in(1.100 e+23)$.

$2 b, \operatorname{In} f(x, y, z): x \in(1.5 e+08), \operatorname{Std} \in(1.155 e+08) ; y \in(-3.584 e+23), \operatorname{Std} \in(3.665 e+23)$.

$3 c$, In $f(x, z, n): x \in(1.5 e+08), \operatorname{Std} \in(1.155 e+08) ; z \in(-1.792 e+24), \operatorname{Std} \in(1.833 e+24)$.

$4 d, \operatorname{In} f(x, y, k): x \in(1.5 e+08), \operatorname{Std} \in(1.155 e+08) ; k \in(-3.584 e+23), \operatorname{Std} \in(3.665 e+23)$.

In the four sets of fitting conclusions, SSE is the sum variance $=0$, indicating that the model fitting prediction is accurate. Comparing the average value Std of the goodness of fit of the structure, contrast $1 ; 2 ; 3 ; 4$. It can be concluded that $\operatorname{Std} \in(1.100 \mathrm{e}+23)$ is the minimum value. We can obtain $1 \mathrm{a}$ as the optimal model.

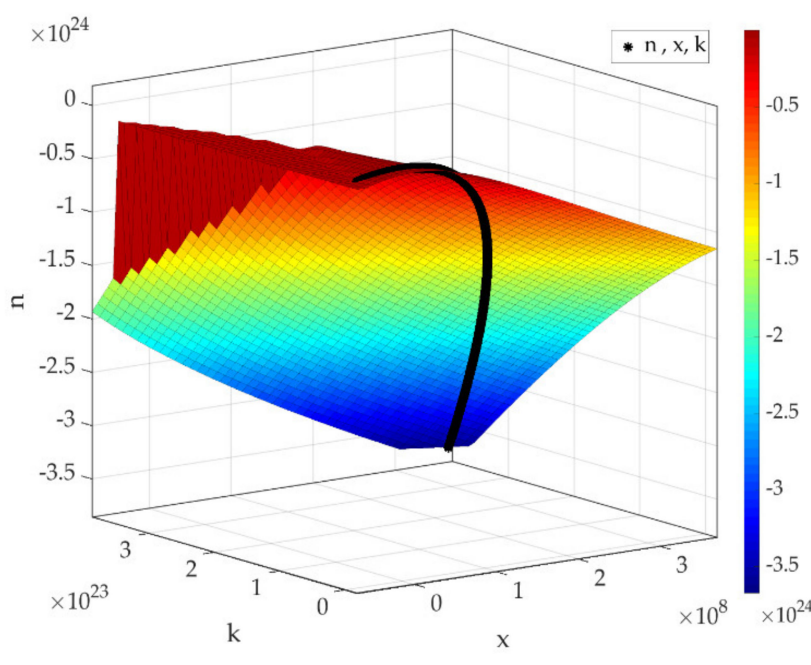

(a)

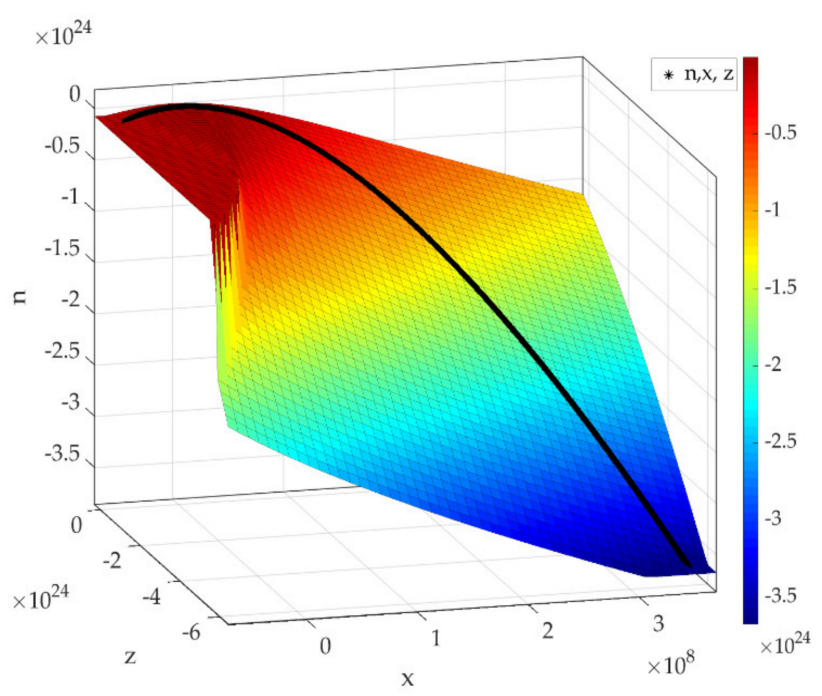

(c)

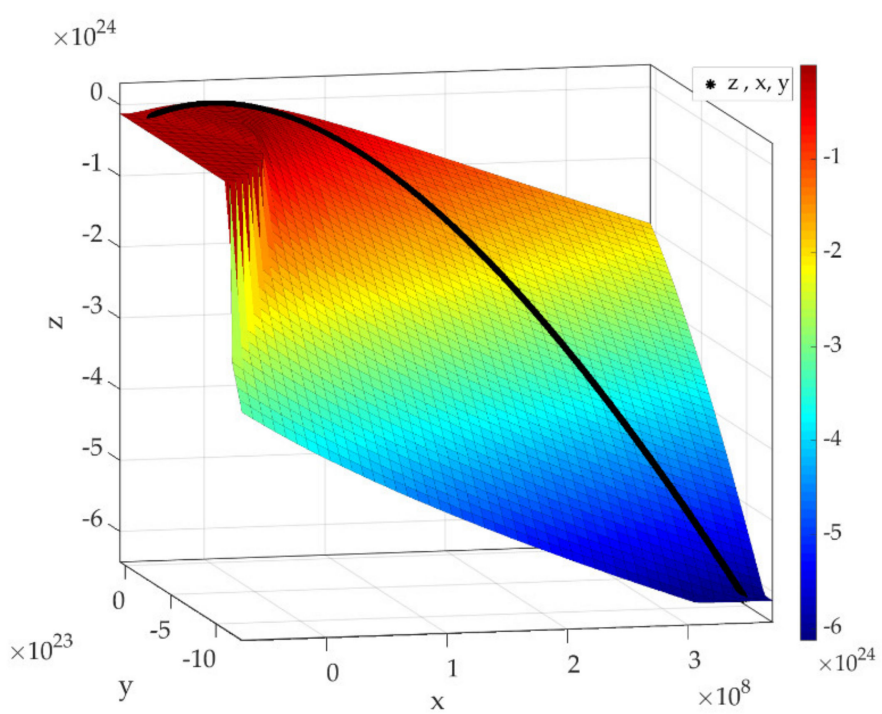

(b)

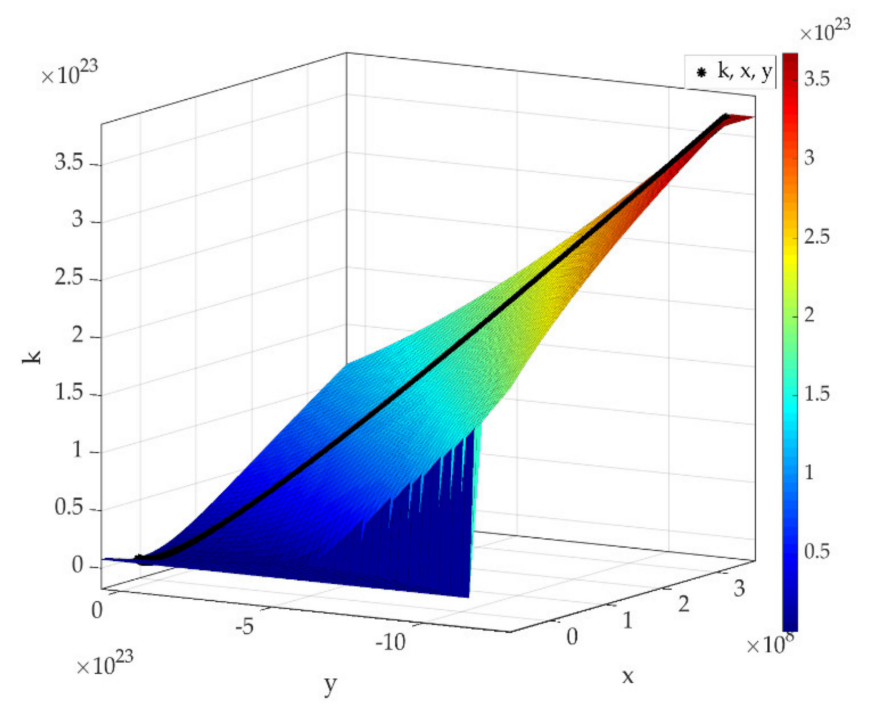

(d)

Figure 17. Nearest neighbor interpolant fitting with four modes: (a) $f(x, k, n) ;(b) f(x, y, z) ;(c) f(x, z, n) ;(d) f(x, y, k)$.

\subsection{Evaluation System Innovation}

Table 6 shows the designed best project management mode indicates a reasonable management plan implemented with the lowest environmental impact and economic cost. 
Table 6. Summary of project management data.

\begin{tabular}{cccc}
\hline Pier Number & 9\# 17\# & 17\# 22\# & 22\# 25\# \\
\hline Structural style & $\begin{array}{c}\text { Prefabricated T-beam, } \\
\text { installed on site. }\end{array}$ & $\begin{array}{c}\text { On-site hanging basket } \\
\text { method construction. }\end{array}$ & $\begin{array}{c}\text { Prefabricated T-beam installed } \\
\text { on site. }\end{array}$ \\
\hline
\end{tabular}

Through the establishment of theoretical framework and case study, it is found that it is necessary to modify the standard performance of the Figure 4 project evaluation system to realize the comprehensive evaluation of the environmental pollution index of the construction industry. Figure 18 shows the optimization and improvement of project management evaluation framework for the whole lifecycle of the construction industry.

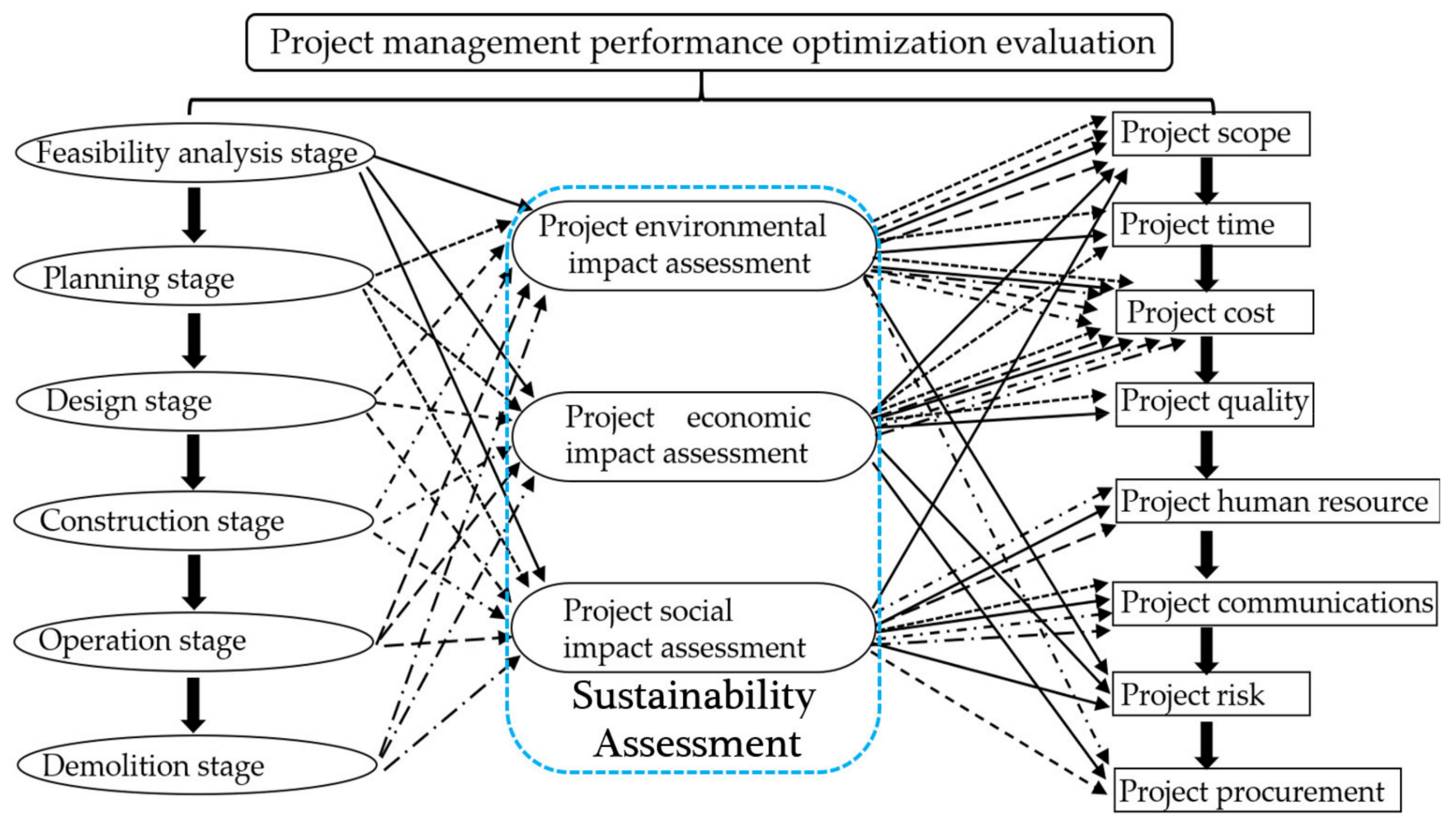

Figure 18. Optimized sustainable development project management evaluation framework system.

\section{Conclusions and Future Trends}

Through the analysis of the published research results, the authors found that the project management framework and model have evaluation flaws. Focus on the evaluation of economic costs, quality and other factors, leads to a lack of focus on the environmental impact assessment system. The existing environmental assessment system exists in the project evaluation stage, survey, and design stage. The environmental assessment of these two stages is designed to meet the requirements of project approval and construction laws and regulations.

Through the project evaluation framework and modeling theory established in this paper, the case study is analyzed. Through the evaluation of the research of the project case construction stage, it is found that the project management mode is superior, which is very important in reducing environmental pollution. The results show that the environmental pollution caused by the construction of ancillary facilities and the main works of the third project management mode is reduced by $62,738.33$ tonnes to $208,766.58$ tonnes compared with the first mode, and the economic cost is reduced by CNY 4.063 million to CNY 6.916 million, realizing high project profit. 
The research results remind structural/construction engineers to fully consider the impact of their project management on sustainable development, and how to reduce the impact by optimizing the design in the design stage; how to evaluate and review its impact. The goal is to build green home with sustainable concepts.

This study is limited to the study area, so future lines of research will diversify the type of bridges, capital investment in sustainable green building innovation; early stage research and development costs of new energy; uneven economic development in various countries around the world, among others. These are all constraints on a clean environment and sustainable development in the world.

The theoretical framework and analysis process of the research fully meet the reference and deepening needs of researchers in the same field. The implementation of sustainable project management is the reform direction and development trend of the global construction industry in the future and it is bound to occupy the construction market; it will attract the attention of governments and international organizations around the world; continuous improvement and implementation are imminent.

Author Contributions: Conceptualization, Z.Z., V.Y. and J.A.; methodology, Z.Z.; software, Z.Z.; validation, J.A., V.Y.; formal analysis, Z.Z. and J.A.; investigation, Z.Z.; resources, J.A. and V.Y.; data curation, Z.Z.; writing—original draft preparation, Z.Z.; writing—review and editing, J.A. and V.Y.; supervision, V.Y.; project administration, J.A.; funding acquisition, V.Y. All authors have read and agreed to the published version of the manuscript.

Funding: This research was funded by the Spanish Ministry of Science and Innovation, along with FEDER (Fondo Europeo de Desarrollo Regional), project grant number: PID2020-117056RB-I00.

Conflicts of Interest: The authors declare no potential conflict of interest with respect to the research, authorship, and/or publication of this article.

\section{References}

1. Stocker, T.F.; Qin, D.; Plattner, G.-K.; Tignor, M.M.B.; Allen, S.K.; Boschung, J.; Nauels, A.; Xia, Y.; Bex, V.; Midgley, P.M. Climate Change 2013-the Fifth Assessment Report on Climate Change Edited; Department of Energy Office of Scientific and Technical Information: Washington, WA, USA, 2013. Available online: https:/ /www.osti.gov/etdeweb/biblio/22221318 (accessed on 18 May 2021).

2. García-Segura, T.; Penadés-Plà, V.; Yepes, V. Sustainable bridge design by metamodel-assisted multi-objective optimization and decision-making under uncertainty. J. Clean. Prod. 2018, 202, 904-915. [CrossRef]

3. Wang, Y.; Liang, S. Carbon dioxide mitigation target of China in 2020 and key economic sectors. Energy Policy 2013, 58, 90-96. [CrossRef]

4. Lin, B.; Liu, $\mathrm{H} . \mathrm{CO}_{2}$ mitigation potential in China's building construction industry: A comparison of energy performance. Build. Environ. 2015, 94, 239-251. [CrossRef]

5. Zhou, Z.; Alcalá, J.; Yepes, V. Environmental, Economic and Social Impact Assessment: Study of Bridges in China's Five Major Economic Regions. Int. J. Environ. Res. Public Health 2020, 18, 122. [CrossRef]

6. Caruso, G.; Gattone, S.; Fortuna, F.; Di Battista, T. Cluster Analysis for mixed data: An application to credit risk evaluation. Socio-Econ. Plan. Sci. 2021, 73, 100850. [CrossRef]

7. D'Adamo, I.; Gastaldi, M.; Morone, P. The post COVID-19 green recovery in practice: Assessing the profitability of a policy proposal on residential photovoltaic plants. Energy Policy 2020, 147, 111910. [CrossRef]

8. Dobrovolskienè, N.; Pozniak, A.; Tvaronavičienè, M. Assessment of the Sustainability of a Real Estate Project Using Multi-Criteria Decision Making. Sustainability 2021, 13, 4352. [CrossRef]

9. Elsevier Scopus. Available online: https://www.elsevier.com/solutions/scopus (accessed on 20 May 2021).

10. Chen, C. CiteSpace: Visualizing Patterns and Trends in Scientific Literature. 2021. Available online: http://cluster.cis.drexel.edu/ $\sim\{\}$ cchen/citespace/ (accessed on 21 May 2021).

11. Invernizzi, D.C.; Locatelli, G.; Brookes, N.J. Characterising nuclear decommissioning projects: An investigation of the project characteristics that affect the project performance. Constr. Manag. Econ. 2020, 38, 947-963. [CrossRef]

12. Yun, L.; Wan, J.; Wang, G.; Bai, J.; Zhang, B. Exploring the missing link between top management team characteristics and megaproject performance. Eng. Constr. Arch. Manag. 2020, 27, 1039-1064. [CrossRef]

13. Elghaish, F.; Abrishami, S. A centralised cost management system: Exploiting EVM and ABC within IPD. Eng. Constr. Arch. Manag. 2020, 28, 549-569. [CrossRef]

14. Owusu-Manu, D.-G.; Debrah, C.; Amissah, L.; Edwards, D.J.; Chileshe, N. Exploring the linkages between project managers' mindset behaviour and project leadership style in the Ghanaian construction industry. Eng. Constr. Arch. Manag. 2020, 1-22. [CrossRef] 
15. Vilventhan, A.; Rajadurai, R. 4D Bridge Information Modelling for management of bridge projects: A case study from India. Built Environ. Proj. Asset Manag. 2019, 10, 423-435. [CrossRef]

16. Liang, R.; Chong, H.-Y. A hybrid group decision model for green supplier selection: A case study of megaprojects. Eng. Constr. Arch. Manag. 2019, 26, 1712-1734. [CrossRef]

17. Yadollahi, M.; Nazari, R.; Spanos, N.J.; Minner, N. An application of fuzzy factor analysis for sustainable bridge maintenance and retrofit projects. Int. J. Manag. Sci. Eng. Manag. 2016, 12, 225-236. [CrossRef]

18. Brockmann, C.; Brezinski, H.; Erbe, A. Innovation in Construction Megaprojects. J. Constr. Eng. Manag. 2016, 142, 04016059. [CrossRef]

19. Ravindra, R.; Fatemi-Nayeri, H. Managing Challenges and Mitigating Risks in Design, Construction, and Product Conformity of the Shared Path Bridge over Epping Road at Marsfield. Pr. Period. Struct. Des. Constr. 2016, 21, 04016002. [CrossRef]

20. Larsson, J.; Lu, W.; Krantz, J.; Olofsson, T. Discrete Event Simulation Analysis of Product and Process Platforms: A Bridge Construction Case Study. J. Constr. Eng. Manag. 2016, 142, 04015097. [CrossRef]

21. Ballesteros-Pérez, P.; Del Campo-Hitschfeld, M.L.; González-Naranjo, M.A.; González-Cruz, M.C. Climate and construction delays: Case study in Chile. Eng. Constr. Arch. Manag. 2015, 22, 596-621. [CrossRef]

22. Gao, T.; Ergan, S.; Akinci, B.; Garrett, J.H. Proactive Productivity Management at Job Sites: Understanding Characteristics of Assumptions Made for Construction Processes during Planning Based on Case Studies and Interviews. J. Constr. Eng. Manag. 2014, 140, 04013054. [CrossRef]

23. Jensen, P.A. Knowledge transfer from facilities management to building projects: A typology of transfer mechanisms. Arch. Eng. Des. Manag. 2012, 8, 170-179. [CrossRef]

24. Williams, T. Identifying Success Factors in Construction Projects: A Case Study. Proj. Manag. J. 2016, 47, 97-112. [CrossRef]

25. Müller, R.; Turner, R. The Influence of Project Managers on Project Success Criteria and Project Success by Type of Project. Eur. Manag. J. 2007, 25, 298-309. [CrossRef]

26. Wilker, J.; Rusche, K.; Rymsa-Fitschen, C. Improving Participation in Green Infrastructure Planning. Plan. Pr. Res. 2016, 31, 229-249. [CrossRef]

27. Shen, L.; Wu, Y.; Zhang, X. Key Assessment Indicators for the Sustainability of Infrastructure Projects. J. Constr. Eng. Manag. 2011, 137, 441-451. [CrossRef]

28. Feng, Q.; Chen, H.; Shi, X.; Wei, J. Stakeholder games in the evolution and development of green buildings in China: Governmentled perspective. J. Clean. Prod. 2020, 275, 122895. [CrossRef]

29. Yu, H.; Solvang, W.D.; Chen, C. A green supply chain network design model for enhancing competitiveness and sustainability of companies in high north arctic regions. Int. J. Energy Environ. 2014, 5, 403-418. Available online: https://www.researchgate.net/ publication/265301217 (accessed on 31 May 2021).

30. Lin, Y.-Q.; Guo, C.-X.; Tan, Y. The incentive and coordination strategy of sustainable construction supply chain based on robust optimisation. J. Control. Decis. 2020, 7, 126-159. [CrossRef]

31. Tayeh, B.A.; Abu Aisheh, Y.I.; Abuzuhri, I.O. Factors Affecting Sustainability Performance during the Construction Stage in Building Projects-Consultants' Perspective. Open Constr. Build. Technol. J. 2020, 14, 17-26. [CrossRef]

32. Meharie, M.G.; Gariy, Z.C.A.; Mutuku, R.N.N.; Mengesha, W.J. Prioritizing Key Duration Estimation Accuracy Factors in Highway Infrastructure Projects Using Fuzzy AHP. Open Civ. Eng. J. 2019, 13, 92-108. [CrossRef]

33. Zhong, Y. Research on Construction Engineering Project Management Optimization Based on C4.5 Improved Algorithm. In Proceedings of the IOP Conference Series: Materials Science and Engineering; IOP Publishing: Bristol, UK, 2019; Volume 688, p. 055036.

34. Baudrit, C.; Taillandier, F.; Tran, T.T.P.; Breysse, D. Uncertainty Processing and Risk Monitoring in Construction Projects Using Hierarchical Probabilistic Relational Models. Comput. Civ. Infrastruct. Eng. 2019, 34, 97-115. [CrossRef]

35. Cho, K.; Hong, T.; Hyun, C. Effect of project characteristics on project performance in construction projects based on structural equation model. Expert Syst. Appl. 2009, 36, 10461-10470. [CrossRef]

36. Kang, Y.; O’Brien, W.J.; Thomas, S.; Chapman, R.E. Impact of Information Technologies on Performance: Cross Study Comparison. J. Constr. Eng. Manag. 2008, 134, 852-863. [CrossRef]

37. PMBOK. PMBOK Guide and Standards Project Management. PA, USA. 2021. Available online: https://www.pmi.org/pmbokguide-standards (accessed on 21 May 2021).

38. Qureshi, T.M.; Warraich, A.S.; Hijazi, S.T. Significance of project management performance assessment (PMPA) model. Int. J. Proj. Manag. 2009, 27, 378-388. [CrossRef]

39. Chou, J.-S.; Yang, J.-G. Project Management Knowledge and Effects on Construction Project Outcomes: An Empirical Study. Proj. Manag. J. 2012, 43, 47-67. [CrossRef]

40. Ling, F.Y.; Low, S.P.; Wang, S.; Egbelakin, T. Models for Predicting Project Performance in China Using Project Management Practices Adopted by Foreign AEC Firms. J. Constr. Eng. Manag. 2008, 134, 983-990. [CrossRef]

41. Zwikael, O. The Relative Importance of the PMBOK ${ }^{\circledR}$ Guide's Nine Knowledge Areas during Project Planning. Proj. Manag. J. 2009, 40, 94-103. [CrossRef]

42. Yeung, J.F.; Chan, A.P.; Chan, D.W. Developing a Performance Index for Relationship-Based Construction Projects in Australia: Delphi Study. J. Manag. Eng. 2009, 25, 59-68. [CrossRef]

43. Hoogmartens, R.; Van Passel, S.; Van Acker, K.; Dubois, M. Bridging the gap between LCA, LCC and CBA as sustainability assessment tools. Environ. Impact Assess. Rev. 2014, 48, 27-33. [CrossRef] 
44. Kang, H.; Lee, Y.; Kim, S. Sustainable building assessment tool for project decision makers and its development process. Environ. Impact Assess. Rev. 2016, 58, 34-47. [CrossRef]

45. Dikmen, I.; Qazi, A.; Erol, H.; Birgonul, M.T. Meta-Modeling of Complexity-Uncertainty-Performance Triad in Construction Projects. Eng. Manag. J. 2021, 33, 30-44. [CrossRef]

46. Haque, T.S.; Chakraborty, A.; Mondal, S.P.; Alam, S. Approach to solve multi-criteria group decision-making problems by exponential operational law in generalised spherical fuzzy environment. CAAI Trans. Intell. Technol. 2020, 5, 106-114. [CrossRef]

47. Ning, W.; Yingbo, J. Analysis on Key Factors to Affect Construction Industrialization Development in China. In Proceedings of the 2010 3rd International Conference on Information Management, Innovation Management and Industrial Engineering, Kunming, China, 26-28 November 2010; Volume 2, pp. 64-67.

48. Pellicer, E.; Yepes, V.; Correa, C.L.; Alarcón, L.F. Model for Systematic Innovation in Construction Companies. J. Constr. Eng. Manag. 2014, 140, 4014001. [CrossRef]

49. De Melo, J.C.F.; Salerno, M.S.; Freitas, J.S.; Bagno, R.B.; Brasil, V.C. From open innovation projects to open innovation project management capabilities: A process-based approach. Int. J. Proj. Manag. 2020, 38, 278-290. [CrossRef]

50. Landi, D.; Marconi, M.; Bocci, E.; Germani, M. Comparative life cycle assessment of standard, cellulose-reinforced and end of life tires fiber-reinforced hot mix asphalt mixtures. J. Clean. Prod. 2020, 248, 119295. [CrossRef]

51. Kim, K.; Lee, G.; Kim, S. A Study on the Application of Blockchain Technology in the Construction Industry. KSCE J. Civ. Eng. 2020, 24, 2561-2571. [CrossRef]

52. Behera, P.; Mohanty, R.; Prakash, A. Understanding Construction Supply Chain Management. Prod. Plan. Control. 2015, 26, 1332-1350. [CrossRef]

53. Navarro, I.; Yepes, V.; Martí, J.V. Social life cycle assessment of concrete bridge decks exposed to aggressive environments. Environ. Impact Assess. Rev. 2018, 72, 50-63. [CrossRef]

54. Mirza, E.; Ehsan, N. Quantification of Project Execution Complexity and its Effect on Performance of Infrastructure Development Projects. Eng. Manag. J. 2017, 29, 108-123. [CrossRef]

55. Laine, T.; Korhonen, T.; Suomala, P. The dynamics of repairing multi-project control practice: A project governance viewpoint. Int. J. Proj. Manag. 2020, 38, 405-418. [CrossRef]

56. Zhou, Z.; Alcalá, J.; Yepes, V. Bridge Carbon Emissions and Driving Factors Based on a Life-Cycle Assessment Case Study: Cable-Stayed Bridge over Hun He River in Liaoning, China. Int. J. Environ. Res. Public Health 2020, 17, 5953. [CrossRef]

57. LaClair, T.J.; Truemner, R. Modeling of Fuel Consumption for Heavy-Duty Trucks and the Impact of Tire Rolling Resistance. SAE Tech. Pap. Ser. 2005. [CrossRef]

58. Sannino, A.; Postiglione, G.; Bollen, M. Feasibility of a DC network for commercial facilities. IEEE Trans. Ind. Appl. 2003, 39, 1499-1507. [CrossRef]

59. Richards, T.J.; Hamilton, S.F. Food waste in the sharing economy. Food Policy 2018, 75, 109-123. [CrossRef]

60. Bai, S.; Zhang, X.; Xiang, Y.; Wang, X.; Zhao, X.; Ren, N. HIT.WATER scheme: An integrated LCA-based decision-support platform for evaluation of wastewater discharge limits. Sci. Total Environ. 2019, 655, 1427-1438. [CrossRef] [PubMed]

61. Jiang, Y.; Zhang, L.; Zhang, J. Energy consumption by rural migrant workers and urban residents with a hukou in China: Quality-of-life-related factors and built environment. Nat. Hazards 2019, 99, 1431-1453. [CrossRef]

62. Frangopol, D.M.; Dong, Y.; Sabatino, S. Bridge life-cycle performance and cost: Analysis, prediction, optimisation and decisionmaking. Struct. Infrastruct. Eng. 2017, 13, 1239-1257. [CrossRef]

63. Bozejko, W.; Hejducki, Z.; Wodecki, M. Flowshop scheduling of construction processes with uncertain parameters. Arch. Civ. Mech. Eng. 2019, 19, 194-204. [CrossRef]

64. Wang, Z.; Yang, D.Y.; Frangopol, D.M.; Jin, W. Inclusion of environmental impacts in life-cycle cost analysis of bridge structures. Sustain. Resilient Infrastruct. 2019, 5, 252-267. [CrossRef]

65. General Code for Design of Highway Bridges and Culverts" (JTG D60-2015). Ministry of Transport of the People's Republic of China: Beijing, China. 2015. Available online: https://www.mot.gov.cn/jiaotongtushu/201512/t20151229_1967723.html (accessed on 16 May 2021). (In Chinese)

66. Dehestani, H.; Ordokhani, Y.; Razzaghi, M. Application of the modified operational matrices in multiterm variable-order time-fractional partial differential equations. Math. Methods Appl. Sci. 2019, 42, 7296-7313. [CrossRef]

67. Martinsuo, M.; Geraldi, J. Management of project portfolios: Relationships of project portfolios with their contexts. Int. J. Proj. Manag. 2020, 38, 441-453. [CrossRef]

68. Panwar, A.; Jha, K.N. A many-objective optimization model for construction scheduling. Constr. Manag. Econ. 2019, 37, 727-739. [CrossRef]

69. Penadés-Plà, V.; Martí, J.V.; García-Segura, T.; Yepes, V. Life-Cycle Assessment: A Comparison between Two Optimal PostTensioned Concrete Box-Girder Road Bridges. Sustainability 2017, 9, 1864. [CrossRef]

70. García-Segura, T.; Yepes, V.; Frangopol, D. Multi-objective design of post-tensioned concrete road bridges using artificial neural networks. Struct. Multidiscip. Optim. 2017, 56, 139-150. [CrossRef]

71. Afshari, A.R. Methods for Selection of Construction Project Manager: Case Study. J. Constr. Eng. Manag. 2017, $143,06017003$. [CrossRef]

72. Wiewiora, A.; Chang, A.; Smidt, M. Individual, project and organizational learning flows within a global project-based organization: Exploring what, how and who. Int. J. Proj. Manag. 2020, 38, 201-214. [CrossRef] 
73. Ministry of Transport, Code for Pile Foundation of Port Engineering (JTJ254-98). Beijing, China. 2020. Available online: http:/ / www.mot.gov.cn/xiazaizhongxin/ziliaoxiazai/201705/P020200709631687274876.pdf (accessed on 18 May 2021).

74. Mathworks Matlab and Training. MA, USA. 2021. Available online: https://matlabacademy.mathworks.com/?s_tid=pl_learn (accessed on 20 May 2021).

75. Zhou, Z.-W.; Alcalá, J.; Kripka, M.; Yepes, V. Life Cycle Assessment of Bridges Using Bayesian Networks and Fuzzy Mathematics. Appl. Sci. 2021, 11, 4916. [CrossRef] 